\title{
Corela
}

Cognition, représentation, langage

HS-9 | 2010

Parole

\section{Les préfixes anglais un- et de- : Étude phonétique et acoustique}

Sylvie Hanote, Nicolas Videau, Franck Zumstein et Philippe Carré

\section{(2) OpenEdition}

\section{Journals}

Édition électronique

URL : https://journals.openedition.org/corela/1081

DOI : 10.4000/corela.1081

ISSN : $1638-573 \mathrm{X}$

Éditeur

Cercle linguistique du Centre et de l'Ouest - CerLICO

Référence électronique

Sylvie Hanote, Nicolas Videau, Franck Zumstein et Philippe Carré, « Les préfixes anglais un- et deÉtude phonétique et acoustique », Corela [En ligne], HS-9 | 2010, mis en ligne le 05 mars 2010, consulté le 18 juin 2021. URL : http://journals.openedition.org/corela/1081 ; DOI : https://doi.org/ 10.4000/corela.1081

Ce document a été généré automatiquement le 18 juin 2021.

\section{c) (1)(2)}

Corela - cognition, représentation, langage est mis à disposition selon les termes de la licence Creative Commons Attribution - Pas d'Utilisation Commerciale - Partage dans les Mêmes Conditions 4.0 International. 


\title{
Les préfixes anglais un- et de- : Étude phonétique et acoustique
}

\author{
Sylvie Hanote, Nicolas Videau, Franck Zumstein et Philippe Carré
}

\section{Introduction}

1 Dans cette étude, nous nous intéressons à la prononciation des deux préfixes un- et deen anglais. On distingue généralement deux types de préfixe : les préfixes séparables et les préfixes inséparables (ou pseudo-préfixes). Les préfixes séparables s'accolent à une base autonome et le sens de l'ensemble est compositionnel de la somme des deux parties. Par exemple, de- dans le verbe debone est séparable puisque bone est une base autonome, et que debone signifie " to remove the bones from », mais il est inséparable dans deceive puisque -ceive n'est pas autonome.

2 En revanche, un- est généralement considéré comme uniquement séparable. C'est le cas par exemple dans unfair où fair est une base autonome et unfair signifie not fair.

À l'oral, la séparabilité du préfixe se traduit normalement par le fait qu'il est porteur d'un accent :

Dans les préfixés à préfixe séparable, le préfixe et le radical sont traités comme des mots indépendants et en suivent les règles d'accentuation et de prononciation. (Guierre, L. 1987: 18)

Il s'agit généralement d'accent secondaire quand le mot est un verbe (to debone /21/) ou un adjectif (unfair /21/), principal lorsque c'est un nom (a decaf /10/). Par ailleurs, la voyelle d'un préfixe séparable a généralement une valeur pleine, relâchée dans le cas de un- $([\Lambda \mathrm{n}])$, tendue dans le cas de de- ([di:]). Dans cette étude, nous nous intéresserons dans un premier temps aux transcriptions par les dictionnaires de ces deux préfixes proposées et aux régularités proposées par L. Guierre. Ensuite, nous comparerons ces transcriptions aux données observables en discours et nous verrons que la prononciation du préfixe peut dépendre d'autres facteurs que celui de la séparabilité / inséparabilité du préfixe et de la base. 


\section{Cadre théorique et notation des préfixes dans les dictionnaires}

4 À partir des considérations théoriques rappelées en introduction, nous avons voulu observer la notation des deux préfixes de- et un- dans les dictionnaires. Nous avons choisi comme corpus de référence le dictionnaire de J.C. Wells, édition de 1990 (désormais LPD1), car il existe sous une forme numérique, enrichie par L. Guierre de données supplémentaires ${ }^{1}$ et de balises, ce qui nous a permis d'effectuer des tris automatiques avec le logiciel de programmation $M P W^{2}$. Grâce à ce logiciel, nous avons pu extraire les mots pertinents pour notre analyse (préfixés en de- et un-) et les trier selon leur schéma accentuel et selon la valeur de la voyelle du préfixe.

Pour ce qui est de l'accentuation des préfixes séparables, on rencontre quatre cas de figure dans le dictionnaire de référence :

- le préfixe peut être inaccentué /0/,

- il peut porter un accent secondaire $/ 2 /$,

- il peut être optionnellement accentué /(2)/,

- il peut porter un accent principal /1/.

\section{Le préfixe un- : notation dans le corpus de référence (LPD1) et les problèmes rencontrés}

5 Dans LPD1, nous avons relevé 493 items préfixés en -un qui se répartissent de la manière suivante :

Figure 1

\begin{tabular}{|l|l|l|l|}
\hline Schéma accentuel 1 & Nombre & Pourcentage & Exemple \\
\hline$/ 0-/$ & 80 & $16.2 \%$ & Uncover, unwillingly, unwieldy \\
\hline$/ 2-/$ & 277 & $56.2 \%$ & Unchecked, unceremonious, unconnected \\
\hline$/(2)-/$ & 136 & $27.6 \%$ & Unnatural, uneducated \\
\hline$/ 1 /$ & 0 & $0 \%$ & \\
\hline
\end{tabular}

6 Nous n'avons trouvé aucun mot préfixé en un- dont la première syllabe porte un accent de rang $/ 1 /{ }^{3}$. En général, les préfixés dans lesquels le préfixe est séparable et reçoit un accent de rang $/ 1 /$ sont les noms de paires d'unités homographiques pluri-catégorielles dans lesquelles un jeu d'opposition accentuelle est en place : re'write (v.) vs. 'rewrite (n.), ,mis'count (v.) vs. 'miscount (n.), ,pre'school (adj.) vs. 'preschool (n.), „pre'script (adj.) vs. 'prescript (n.), etc. Ainsi, les résultats indiqués dans la dernière colonne du tableau ci-dessus indiquent-ils qu'il n'existe aucune paire avec alternance accentuelle incluant un préfixé nominal en un- accentué /1/ sur la première syllabe. Nous avons trouvé 277 mots qui portent un accent secondaire sur le préfixe, dont 157 avec un autre accent (principal ou tertiaire) sur la syllabe suivante (unchecked /21/, unceremonious /23010/), sachant que pour les autres (en /20-/: unconnected /2010/, unconditional /20100/), la 
présence de l'accent secondaire n'est pas pertinente pour rendre compte du statut séparable du préfixe, car l'accent secondaire peut également être interprété comme imposé par la contrainte rythmique ${ }^{4}$. À côté de ce sous-ensemble, 136 mots présentent un accent secondaire optionnel /(2)/. C'est le cas par exemple de unnatural $/(2) 100 /$ ou de uneducated $/(2) 1000 /)^{5}$. Cette présentation d'accent «optionnel» n'est pas un problème de fréquence d'occurrences. Si c'était le cas, le dictionnaire présenterait les choses en terme d'accentuation $n^{\circ} 1 /$ accentuation $n^{\circ} 2$, comme pour des mots comme unusually (schéma accentuel $n^{\circ} 1: / 0100 /$; schéma accentuel $n^{\circ} 2: / 2100 /$ ), nous $y$ reviendrons plus bas. Il ne s'agit donc pas de fréquence d'occurrence mais plutôt d'indétermination, comme le souligne J.C. Wells dans l'entrée consacrée au préfixe (1990 : 744) :

[The prefix un-] is stressed particularly (a) where the initial syllable of the stem does not bear the primary stress (,una'shamed), and (b) in verbs (,un'coil). In some words usage is divided or uncertain ${ }^{6}\left({ }_{1}\right)$ un'bearable).

7 A côté de ces premiers sous-ensembles viennent les mots dans lesquels le préfixe unest inaccentué en prononciation principale (accentuation /01-/). 80 mots font partie de ce sous-ensemble, soit un peu plus de $16 \%$ du corpus. C'est le cas de mots comme uncover, unwillingly, unwieldy. Cette inaccentuation du préfixe est prévue par LPD1 (1990: 744) : "The prefix un- may be lexically stressed or unstressed. It is unstressed particularly when it is not a true prefix (un'wieldy)».

8 Tout d'abord, nous pouvons légitimement nous poser la question du statut du préfixe un- dans le mot unwieldy. En effet, l'adjectif wieldy existe dans le sens « easy to wield or handle; manageable, handy " et unwieldy " difficult to wield, control, or manage, esp. owing to size, shape, or weight; unmanageable ». De ce fait, un- ne peut-il pas être envisageable comme un vrai préfixe ici? Et, s'il est envisageable comme préfixe séparable, pourquoi ne porte-t-il pas d'accent secondaire?

D'autre part, pourquoi le préfixe n'est-il pas accentué dans des mots comme uncover, unfold, ou unwillingly (schéma accentuel /01-/7) alors qu'il l'est dans des mots comme uncrowned, unused, unskilled ou unquote (notés /21/ dans le dictionnaire) ou encore dans des mots comme undo, unearth, unleash, unload qui portent un accent secondaire optionnel sur le préfixe (schéma /(2)1/) ? Quelle est la différence de formation de ces mots? Un- n'est-il pas systématiquement associé à une base sémantiquement autonome (cover, fold, skilled, do, load, etc.) ? Dans EPD15, les auteurs donnent comme seule explication la note suivante :

Un- may be unstressed, usually when a stressed syllable follows it and it is a frequently-used word, e.g. unable / $\Lambda$ n'erbl/ . Otherwise it will have secondary stress, e.g. unadvisable /,^nəd'vaIzəbll/.

Pour unadvisable $e^{8}$ la présence de l'accent secondaire peut être envisagé comme liée aux contraintes rythmiques, donc non pertinente pour l'analyse. Ensuite, pour ce qui est de l'argument qui relie l'absence d'accent à la fréquence d'occurrence, tient-il vraiment pour des mots tels que unfair, unlike, unseen, etc., qui portent un accent de rang /2/ sur le préfixe ? Sont-ils moins fréquemment employés que unable /010/?

11 Ainsi, au vu du corpus, il semble difficile de faire ressortir des contraintes claires quant à la présence / absence d'accent sur le préfixe dans le dictionnaire de référence, LPD1. Par ailleurs, dans ce même dictionnaire est noté un phénomène de variation mentionné plus haut qu'il est important de prendre en considération dans cette étude mais qui semble également aléatoire. En effet, si l'on reprend les 80 mots répertoriés en /01-/ 
par exemple, 41 (soit plus de $50 \%$ ), présentent une variante avec accent secondaire sur le préfixe.

Figure 2

\begin{tabular}{|l|l|l|l|l|l|}
\hline Mot & \multicolumn{2}{|l|}{ Schéma accentuel } & Dérivant & \multicolumn{2}{l|}{ Schéma accentuel } \\
\hline & Principal & Secondaire & & Principal & Secondaire \\
\hline unusually & $/ 010(0) 0 /$ & $/ 210(0) 0 /$ & unsusual & $/ 010(0) /$ & $/ 210(0) /$ \\
\hline uncertainty & $/ 0100 /$ & $\varnothing$ & uncertain & $/ 010 /$ & $/ 210 /$ \\
\hline uneasy & $/ 010 /$ & $/ 210 /$ & unease & $/ 01 /$ & $/ 21 /$ \\
\hline unpleasantness & $/ 0100 /$ & $\varnothing$ & unpleasant & $/ 010 /$ & $\varnothing$ \\
\hline unwillingly & $/ 0100 /$ & $\varnothing$ & unwilling & $/(2) 10 /$ & $\varnothing$ \\
\hline
\end{tabular}

13 Des paires dérivationnelles telles que unusual > unusually et unease > uneasy montrent que la neutralité de la dérivation se traduit par un isomorphisme accentuel strict, tant pour les formes accentuelles principales que pour les formes accentuelles en variantes. Ce n'est pas le cas, en revanche, pour les paires uncertain > uncertainty et unwilling > unwillingly. Pourquoi ne trouve-t-on pas les mêmes variantes pour le dérivé et pour le dérivant $^{9}$ ? Là encore, il semble difficile de dégager des critères généraux ou spécifiques qui ont guidé les choix accentuels pour le préfixe.

14 Ainsi, si l'accentuation peut être un indice précieux dans la détermination du statut du préfixe, de par la variation qui existe, elle ne peut sans doute pas être retenue comme un critère essentiel et stable.

\section{Les critères segmentaux : Valeur de la voyelle du préfixe}

Les observations faites dans la section précédente montrent que la notation de l'accentuation du préfixe séparable un- n'est pas systématique dans LPD1, même si c'est majoritairement le cas. Par contre, il est important de noter que le préfixe, accentué ou non, ne subit pas de réduction de sa voyelle ; il est en effet toujours noté $[\Lambda \mathrm{n}]$. A propos de la valeur de la voyelle du préfixe séparable, L. Guierre (1979 : 358-359) notait la chose suivante :

Que l'accentuation soit /11/,/10/ ou /01/, la réduction de la voyelle ne se produit ni en pré-tonique, ni en post-tonique: uncut $/ 11 /, / 10 /, / 01 /$ est toujours $[\Lambda n k \Lambda t]$ unknot $/ 11 /$ ou $/ 01 /$ se prononce toujours [ $[\mathrm{nnnt}]$

16 Seuls deux mots dont la séquence initiale est un- ont une voyelle réduite. Il s'agit de until [ən'tIl] et de unless [ən'les]. En première approximation, on peut deviner que until n'inclut pas le sens négatif de un- et qu'il s'agit donc d'un autre préfixe. Un aperçu de l'étymologie du mot montre qu'en effet, la préforme un- ici est héritée du MoyenAnglais, empruntée au scandinave, et son sens est " as far as, up to ». En revanche, la notion de restriction (" except ») qui est encodée dans le sens de unless en fait un bon candidat, abstraction faite de sa prononciation principale, pour entrer dans la liste des 
préfixés en un-. Néanmoins, l'étymologie du mot indique qu'il s'agit d'une forme altérée de la préforme on : on lesse, à l'origine --> 1438 onlesse --> 1467 unlesse. C'est plus tard, comme l'indique l'OED, que ce préfixe a pu être assimilé au préfixe un-. Néanmoins, il n'en a pas pris les atours phonétiques pour ce qui est de la voyelle, laissant ainsi transparaître ses origines. J.C. Wells signale quand même la variante accentuelle en / (2)1/ pour chacun des deux mots. Du coup, la voyelle est prononcée $[\Lambda]$.

Dans le cas de unless, Wells (1991: 750) fait remarquer que cette accentuation peut apparaître dans une forme emphatique ${ }^{10}$ :

unless ən 'les $\Lambda \mathrm{n}$ - -occasionally also, for emphasis,,$\Lambda \mathrm{n}$ -

17 C'est certainement le cas également, pour until qui, en outre, peut avoir, selon LPD1, une finale désaccentuée, prononcée [-təl]. Dans ce dernier cas, on aboutit à un schéma /20/ $\left(/, \Lambda \mathrm{t}^{\ominus} \mathrm{l} /\right)$ ou $/ 00 /\left(/ \mathrm{nt}^{\ominus} \mathrm{l} /\right)$, tout comme les formes grammaticales en discours ${ }^{11}$. Enfin, ces variantes sont peut-être aussi le résultat d'une remotivation de l'initiale dans l'expression relativement fréquente unless and until que Fowler (1996: 809) décrit comme suit :

Unless and until. A modern 'stengthened' extension of until (the words 'unless and' are often omissible without discernible loss of meaning).

Nous verrons plus loin dans cette étude si la prononciation de un- en forme pleine est toujours respectée en discours.

\section{L'assimilation}

Dans le corpus de référence de L. Guierre ${ }^{12}$, aucune des entrées des mots préfixés en unne présente une assimilation phonétique de la consonne [n] devant les consonnes [p, b, $\mathrm{m}, \mathrm{k}, \mathrm{g}$ ] en première prononciation. Il est certainement possible de conclure ici que ce blocage est l'une des caractéristiques des vrais préfixés. Cependant, s'il n'y a aucune trace d'assimilation en prononciation principale dans la plupart des entrées, J.C. Wells propose les transcriptions de formes assimilées en variante. Seuls quelques mots, regroupés dans le tableau ci-dessous, ne présentent pas cette variante dans leur entrée :

Figure 3

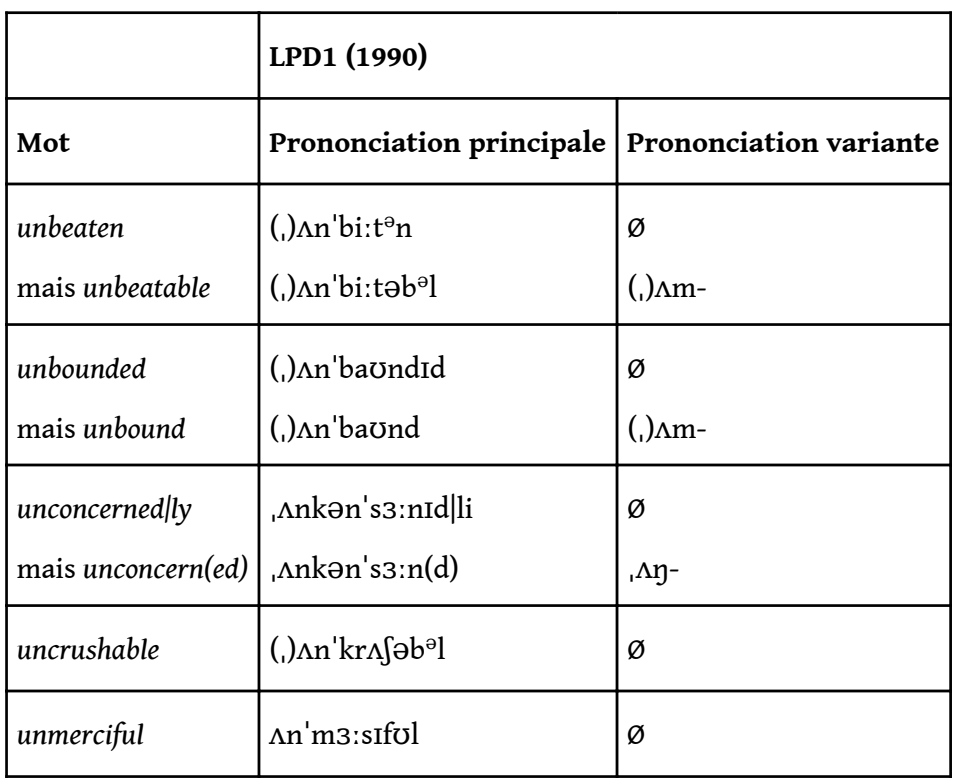




\begin{tabular}{|c|c|c|}
\hline unparalleled & (,) $\Lambda$ n'pærəleld & $\varnothing$ \\
\hline unpunctuality & $, \Lambda \mathrm{n}, \mathrm{p} \Lambda \eta \mathrm{kt} \int \mathrm{u}^{\prime}$ Æləti & $\varnothing$ \\
\hline unpunished & $\left({ }_{1}\right) \wedge n^{\prime} p \wedge n I f t$ & $\varnothing$ \\
\hline
\end{tabular}

\section{Figure 4}

\begin{tabular}{|l|l|l|l|}
\hline \multicolumn{4}{|l|}{ Mots en [dI] } \\
\hline Schéma acc. & Nombre & Pourcentage & Exemples \\
\hline$/ 0-/$ & 339 & $2,2 \%$ & deceive, deform, dethrone... \\
\hline$/ 1-/$ & 0 & $0 \%$ & \\
\hline$/ 2-/$ & 0 & $0 \%$ & \\
\hline$/(2)-/$ & 0 & $0 \%$ & \\
\hline
\end{tabular}



voyelle tendue [di:] est porteur d'un accent secondaire dans $68 \%$ des cas, ou porteur d'un accent secondaire optionnel, dans $21,2 \%$ des cas.

Figure 5

\begin{tabular}{|l|l|l|l|}
\hline \multicolumn{2}{|l|}{ Mots en [di:] } \\
\hline Schéma acc. & Nombre & Pourcentage & Exemples \\
\hline$/ 0-/$ & 4 & $2,2 \%$ & deforestation, defibrilation \\
\hline$/ 1-/$ & 15 & $8,1 \%$ & decoke (noun), devious, demarcate \\
\hline$/ 2-/$ & 125 & $68 \%$ & decompression, decelerate, decrease \\
\hline
\end{tabular}
du verbe deform. En effet, la base form est bel et bien attestée en anglais. Mais, bien que le préfixe ait un sens «négatif » dans deform, il faut analyser le degré de négation dont il est porteur et observer sur quel plan il opère : deform ne veut pas dire « to remove the form of something ", mais " to change the usual shape of something so as to spoil its appearance or usefulness" (définition donnée par le Longman Dictionary of English Language and Culture). Le sens négatif du préfixe est bien présent dans la définition (spoil), mais il ne s'agit ni d'inversion, ni de privation. Ce décalage sémantique semble donc se traduire à l'oral par l'absence d'accent sur le préfixe et par la présence d'une voyelle courte. On notera toutefois que la prononciation [di:] accentuée /21/ apparaitt en variante. plus est, privatif. Pourquoi ce mot n'est-il donc pas accentué en /21/ comme les autres mots à préfixe séparable ? Là encore, un élément potentiel de réponse nous est donné par la définition du mot : «to remove a king or queen from the throne ", et non pas « to remove thrones ». La privation porte donc sur l'argument interne du verbe (l'objet direct, king/queen) et non pas sur la base (throne) $)^{13}$. Cependant, cette hypothèse ne fonctionne pas pour les verbes detrain et derail. En effet, l'oxford Dictionary of English donne les définitions suivantes :

- detrain: « to alight from a railway train » (et non pas « to remove trains »).

- derail: « to run off or leave the rails » (et non pas « to remove rails »).

On pourrait donc s'attendre à un préfixe inaccentué à voyelle courte pour ces deux mots. Or, ce n'est pas le cas puisque le préfixe est porteur d'une voyelle tendue dans les deux mots, et qu'ils sont respectivement accentués en $/ 21 /$ et $/(2) 1 /$. Il est intéressant de noter que le schéma /01/ avec voyelle courte relâchée sur le préfixe apparaît dans l'OED, mais n'est donné en variante dans LPD que pour derail. L'absence de variante en / 01/ pour detrain nous semble problématique. On pourra néanmoins supposer que cette absence est due à la fréquence d'usage de ce verbe, sans doute moins employé que derail (pour exemple, il n'y a pas d'entrée pour detrain dans le Longman Dictionary of English Language and Culture).

comme le prévoit le cadre théorique, le préfixe de-, lorsqu'il est prononcé avec la 


\begin{tabular}{|l|l|l|l|}
\hline$/(2)-/$ & 39 & $21,2 \%$ & decrypt, delimit \\
\hline Autre & 1 & $0,5 \%$ & de- \\
\hline
\end{tabular}

N.B. La catégorie « Autre », qui contient un seul item, correspond à l'entrée du préfixe de-, dont le schéma accentuel n'est pas défini dans le corpus.

Les quatre mots accentués en /0-/ sont des mots en -ation de 5 syllabes ou plus (defibrilation, defoliation, deforestation, dehumanization). L'accent principal tombe sur la pénultième et l'accent secondaire sur la première syllabe de la base. Il est toutefois étrange que ces quatre mots, dans lesquels de- est séparable, ne présentent pas un schéma /23-/ comme c'est le cas pour tous les autres mots de cinq syllabes ou plus en ation (32 items: decomposition, decriminalization, etc.). Notons que l'analyse du cycle dérivationnel de ces mots ne semble pas pouvoir expliquer l'absence d'accent tertiaire dans ces quatre noms. En effet, deforestation /02010/ ne peut être analysé que par l'ajout du suffixe -ation à la base verbale deforest /210/. Le nom *forestation n'est pas attesté, ce qui rejette d'emblée l'analyse par préfixation de-+ forestation. En revanche, le nom defibrillation /02010/ est le résultat de l'ajout du préfixe de-au nom fibrillation ${ }^{14}$, et non d'une suffixation en -ation au verbe defibrillate. De même, on trouve ces deux variétés de cycle dérivationnel parmi les 32 items en /23-/: deregulation (de- + regulation) et decortication (decorticate +-ation).

Les 15 items accentués en /1-/ correspondent à 7 noms dissyllabiques (decoke) et 8 mots à terminaisons contraignantes (devious, demarcate). Il est intéressant de noter que demarcate enfreint la laxing rule qui prédit un préfixe prononcé [de]. Une explication possible est donnée par l'étymologie du mot: l'OED le donne en effet comme étant rétro-formé à partir du nom demarcation, dans lequel le préfixe est prononcé [di:] et porteur d'un accent secondaire. En revanche, rien ne nous permet d'expliquer la voyelle tendue du préfixe dans demarcation.

Dans les verbes decelerate /2100/ et decrease /21/, le préfixe est attaché à une base non autonome (puisque ${ }^{*}$ creas $e^{15}$ et ${ }^{*}$ celerate n'existent pas). Pourtant, le préfixe est bien porteur d'un accent secondaire, et doté d'une voyelle longue.

31 Dans le cas de decelerate, tous les autres dictionnaires interrogés (OED, EPD, Longman Dictionary of English Language and Culture) ont donné le même résultat. La prononciation du préfixe en [dI], prédite par le cadre théorique utilisé n'apparaît qu'en variante dans EPD. Quant au verbe decrease, les dictionnaires le donnent accentué en /01/ avec une voyelle courte, c'est-à-dire la prononciation que l'on peut observer en variante dans LPD. Pour le verbe decrease, nous avons donc supposé que la présence de l'accent /2/ et de la voyelle tendue sur le préfixe s'explique par le fait que le préfixe de-entre dans un paradigme avec le préfixe in- dans increase. En ce qui concerne le verbe decelerate, l'étymologie donnée par OED apporte un élément de réponse : le verbe decelerate vient de de- + (ac)celerate. Le préfixe de- peut donc être ici interprété comme séparable. L'analyse d'un contraste paradigmatique avec le préfixe ac-, allomorphe du préfixe ad-, $\mathrm{du}$ verbe accelerate, n'est pas pour autant à écarter, comme le montre l'analyse acoustique présentée en 3.2.

En ce qui concerne delimit /(2)10/, la base limit est bel et bien autonome en anglais, mais d'autres dictionnaires (OED, Longman Dictionary of English Language and Culture) donnent ce mot avec un préfixe inaccentué (schéma /010/) et à voyelle courte, ce qui semble 
justifié puisque le sens n'est pas compositionnel. En effet, delimit ne veut pas dire « to remove the limits»; il s'agit plutôt ici d'un sens que l'on pourrait qualifier d'« intensif », et que l'on retrouve également dans des mots comme declaim, denude ou defraud dont le préfixe est inaccentué.

Dans les tris faits avec MPW, on trouve également 101 mots dans lesquels le préfixe se prononce [de]. Le préfixe est alors porteur, soit d'un accent primaire, soit d'un accent secondaire.

Figure 6

\begin{tabular}{|l|l|l|l|}
\hline \multicolumn{4}{|l|}{ Mots en [de] } \\
\hline Schéma acc. & Nombre & Pourcentage & Exemples \\
\hline$/ 0-/$ & 0 & $0 \%$ & \\
\hline$/ 1-/$ & 54 & $54,5 \%$ & defecate, deputy \\
\hline$/ 2-/$ & 46 & $46,5 \%$ & debauchee, declaration \\
\hline$/(2)-/$ & 0 & $0 \%$ & \\
\hline
\end{tabular}

Les mots accentués en /1-/ sont de manière générale des mots à terminaisons contraignantes (mots de 3 syllabes en -ate) tels que defecate ou detonate, ou des mots dans lesquels de- semble ne plus être perçu comme un préfixe (deputy, desert).

En ce qui concerne les items présentant un schéma en $/ 2-/$, il s'agit de mots de 3 syllabes ou plus, où l'accent secondaire obéit aux contraintes rythmiques de l'anglais (schéma /201-/ : debauchee, declaration, demolitionist, etc.). Il est intéressant de noter que debauchee présente une variante en /021/ en [dI], très probablement due à son dérivant debauch accentué en /01/.

En ce qui concerne le préfixe diphtongué [deI] (11 items), les différents schémas accentuels possibles sont répartis de manière quasi-égale :

Figure 7

\begin{tabular}{|l|l|l|l|}
\hline \multicolumn{2}{|l|}{ Mots en [deI] } \\
\hline Schéma acc. & Nombre & Pourcentage & Exemples \\
\hline$/ 0-/$ & 4 & $36 \%$ & $\begin{array}{l}\text { debacle [deI'ba:kəl] } \\
\text { declasse [deI'klæseI] } \\
\text { decollete [deI'kplteI] } \\
\text { denouement [deI'nu:mõ] }\end{array}$ \\
\hline$/ 1-/$ & 3 & $27,5 \%$ & $\begin{array}{l}\text { debut ['derbju:] } \\
\text { demarche ['deIma:[] } \\
\text { detente ['deItDnt] }\end{array}$ \\
\hline
\end{tabular}




\begin{tabular}{|l|l|l|l|}
\hline$/ 2-/$ & 3 & $27,5 \%$ & $\begin{array}{l}\text { decolletage [,deIkol'ta:3] } \\
\text { decoupage [,deIku'pa:3] } \\
\text { degage [,deIga:'3eI] }\end{array}$ \\
\hline$/(2)-/$ & 1 & $9 \%$ & demode [(,)deI'məvdeI] \\
\hline
\end{tabular}

La totalité de ces mots sont des emprunts au français. Le corpus inclut par ailleurs la graphie avec accent aigu sur le préfixe. Le problème qui se pose ici est le suivant: les différents schémas accentuels obtenus ne peuvent témoigner d'une quelconque reconnaissance morphologique. En effet, il est plus qu'improbable qu'en anglais, on puisse, par une analyse morphologique et sémantique, déterminer si de- est séparable ou inséparable dans ces emprunts aux français. Ainsi, le fait que le préfixe soit séparable ou non en français n'a aucune influence sur la présence ou l'absence d'accent sur le préfixe en anglais.

Il est toutefois possible d'expliquer la présence de l'accent secondaire sur le préfixe dans les trois items decolletage, decoupage et degage. Ces trois mots sont des mots trisyllabiques recevant un accent principal sur la dernière syllable. Par conséquent, l'accent secondaire sur le préfixe s'explique par les contraintes rythmiques de l'anglais. Enfin, le corpus comporte quatre mots dans lesquels le préfixe est prononcé [di].

Figure 8

\begin{tabular}{|l|l|l|l|}
\hline \multicolumn{2}{|l|}{ Mots en [di] } \\
\hline Schéma acc. & Nombre & Pourcentage & Exemples \\
\hline$/ 0-/$ & 4 & $100 \%$ & deactivate, deactivation, deodorant, deodorize \\
\hline$/ 1-/$ & 0 & $0 \%$ & \\
\hline$/ 2-/$ & 0 & $0 \%$ & \\
\hline$/(2)-/$ & 0 & $0 \%$ & \\
\hline
\end{tabular}

Cette prononciation apparaît toujours à l'inaccentuée et devant une base commençant par une voyelle. En revanche, l'inverse n'est pas systématique, puisque dans des mots tels que de-ice ou deoxygenate, le préfixe est porteur d'un accent secondaire et doté d'une voyelle tendue. Parmi ces quatre mots, seul le couple deactivate / deactivation présente une variante en [di:], alors que le couple deodorize / deodorant ne présente aucune variante sur le préfixe, très probablement parce qu'il est considéré comme inséparable. Il faut toutefois noter que les autres dictionnaires consultés (OED, Longman Dictionary of English Language and Culture) donnent ce dernier couple accentué secondairement sur le préfixe avec une voyelle tendue.

39 A travers l'étude de la notation des préfixes un- et de- dans LPD1, on peut voir que le critère morpho-sémantique ne fonctionne pas dans tous les cas. En effet, la corrélation préfixe séparable $<>$ accent /2/ n'est pas toujours respectée. Ceci peut être lié au fait que la prononciation des préfixes, tant sur le plan suprasegmental que sur le plan 
segmental est liée au contexte dans lequel ces préfixés s'insèrent dans le discours, comme le suggère L. Guierre $(1979: 358)$ selon lequel « les préfixés [à préfixe séparable] ont très couramment une ou des variantes en /10/ ou /01/ contextuellement déterminées à un niveau supérieur à celui du mot ». Cette remarque de Lionel Guierre est très importante, et sera le point de départ de notre troisième partie, dans laquelle nous avons entrepris de mener des analyses acoustiques de préfixés en discours afin de nous focaliser sur des phénomènes dont les dictionnaires ne peuvent rendre compte.

\section{Corpus et outils}

\section{Corpus}

Le corpus oral utilisé pour notre étude acoustique est constitué de documents audio provenant de trois sources différentes.

\section{Le CD-ROM du LPD3 de J.C. Wells (2008)}

Ce CD-ROM comprend des enregistrements de locuteurs britanniques et américains qui lisent la transcription de mots isolés, telle qu'elle est proposée par le dictionnaire. Les problèmes éventuels de ce type de corpus sont les suivants :

- Contraintes et modalités de l'enregistrement (mots prononcés de façon isolée donc pas de prise en compte du contexte)

- Problème parfois car la transcription ne correspond pas à l'enregistrement. Cela est par exemple le cas du mot decrease.

En effet, le verbe decrease est noté comme étant prononcé avec un [i:] en première syllabe avec un accent secondaire sur le préfixe, bien que ce soit un préfixe inséparable : transcription donnée par le dictionnaire : [,di:'kri:s]. On a effectivement sans doute une voyelle tendue [i] (formants identiques à ceux de la voyelle sous accent : $\mathrm{F} 1=407 \mathrm{~Hz}, \mathrm{~F} 2=2093 \mathrm{~Hz}$ ), mais il ne semble pas que ce soit une voyelle longue d'après l'analyse sous Praat (durée de la voyelle du préfixe $=0,06 \mathrm{~s}$ alors que durée de [i: dans la syllabe qui suit $=0,12 \mathrm{~s}$. (Notons qu'il est sans doute difficile de dissocier la liquide $[\mathrm{r}] \mathrm{de}$ la voyelle qui suit dans la deuxième syllabe).

Fichier audio « Decrease LPD » : à écouter à la fin de cet article ...

Figure 9

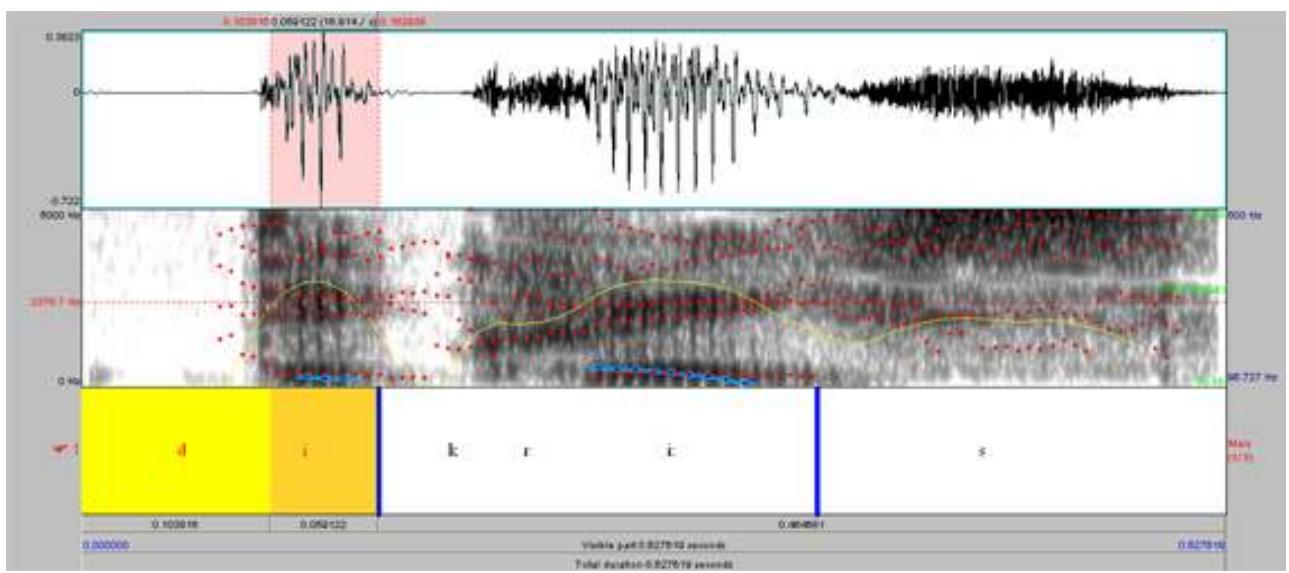




\section{Le corpus « authentique »}

43 l'anglais britannique, et de NPR (National Public Radio) pour l'anglais américain. L'avantage d'un tel corpus est qu'il reflète l'usage de la langue en discours, telle qu'elle est parlée par les locuteurs natifs, ceux-ci étant évidemment non conscients qu'ils feront l'objet d'une étude linguistique. Par contre, la récolte d'items pertinents pour l'étude (préfixés en de- et un-) peut s'avérer longue et fastidieuse, même si le travail est rendu plus aisé aujourd'hui car de nombreuses émissions de ces deux radios sont transcrites orthographiquement.

\section{Le corpus « lecture »}

Nous aurons enfin recours à un corpus "lecture ", qui se compose d'enregistrements effectués au studio Parole de la MSHS de l'Université de Poitiers, où des locuteurs natifs ont été invités à lire une série d'énoncés (issus du BNC) dans lesquels se trouvaient des mots préfixés. L'avantage majeur de ce type de corpus est qu'il est très facile d'obtenir des enregistrements de très bonne qualité de n'importe quel mot dans des contextes précis (par exemple, decelerate isolé dans un énoncé, ou associé à accelerate dans le cotexte proche, nous y reviendrons plus bas). En revanche, ce corpus présente l'inconvénient de ne pas être authentique car bien qu'au moment de la lecture, les locuteurs ne sachent pas sur quelle partie du discours porte l'étude, certains facteurs psycho-linguistiques peuvent entrer en jeu (conscience d'être l'objet d'une étude phonologique, gêne par rapport à la présence d'un micro, etc.) et altérer leur prononciation.

\section{Outils utilisés : Praat et Speech Wave Analyser}

Pour l'analyse acoustique des mots préfixés, nous utilisons deux logiciels de traitement de la parole: le logiciel Praat et le logiciel Speech Wave Analyser développé dans le laboratoire X-LIM-SIC de Poitiers en collaboration avec le laboratoire FORELL.

Le logiciel Praat ${ }^{16}$ est un outil qui a été développé par P. Boersma et D. Weenink (The Institute of Phonetic Sciences, Amsterdam). C'est un logiciel qui permet de mener des analyses phonétiques et acoustiques. Il a l'avantage d'être gratuit, robuste, et de proposer les différentes analyses standards que l'on est susceptible d'utiliser dans le cadre d'une analyse acoustique. De plus il fonctionne sous tous les principaux systèmes d'exploitation (MacOS, Windows, Linux). Il est enfin assez facile d'utilisation et toujours en évolution ; sa toute dernière version Praat 5103 date en effet de mars 2009.

Cependant, bien que Praat constitue un outil d'analyse assez précis, différentes limites peuvent apparaître durant une analyse acoustique fine. Tout d'abord, le module central de Praat utilise des techniques, certes standard, mais issues d'anciennes recherches en traitement du signal, à savoir une analyse Temps-Fréquence suivant le principe de Fourier à fenêtre. Cette analyse consiste en un découpage du signal suivant des intervalles de tailles fixes. Ce découpage est ensuite suivi d'une analyse fréquentielle sur chacun des intervalles, afin d'en extraire une mesure fréquentielle locale. L'élément limitatif est le caractère rigide de la taille des fenêtres temporelles d'analyse. Afin de contourner ceci, depuis plusieurs années, l'analyse du signal a connu de nombreuses 
modifications, débouchant sur des théories très prometteuses, notamment pour l'analyse acoustique.

La seconde limite concerne l'aspect outil «pré-formaté ». Il n'est en effet pas toujours aisé d'avoir une connaissance exacte de l'influence de certains réglages du logiciel et des choix effectués lors du "portage informatique " de ce type de méthode. C'est le concept de boite noire qui peut être gênant ici car cela risque de mettre en doute la fiabilité des résultats des analyses et poser la question de la reproduction et de la confrontation des expérimentations.

Le second outil présenté ici, que nous avons nommé Speech Wave Analyser (désormais SWA), est un outil de traitement de signal basé sur la méthode des ondelettes. L'intérêt présenté par cette méthode réside dans les possibilités de représentation et de découpage du signal dans un espace de représentation temporel/fréquentiel adaptatif.

En effet, ces dernières années, les évolutions majeures de la technique des ondelettes ont porté sur les qualités de partage de l'espace temps-fréquence et sur la recherche d'une solution optimale permettant de mieux segmenter les composantes énergétiques d'un signal dans ce plan. Ces évolutions apportées permettent d'aboutir à une décomposition simple et efficace du signal de parole suivant les phrases, mots, phonèmes ou sons, mais aussi au découpage de bruits assemblés ou de mélodie. L'analyse qui résulte propose des segments de tailles différentes s'adaptant aux composants le plus justement possible, et permettant ainsi une grande précision dans la mesure des fréquences.

Nous proposons d'illustrer cette différence sur un exemple synthétique.

Figure 10

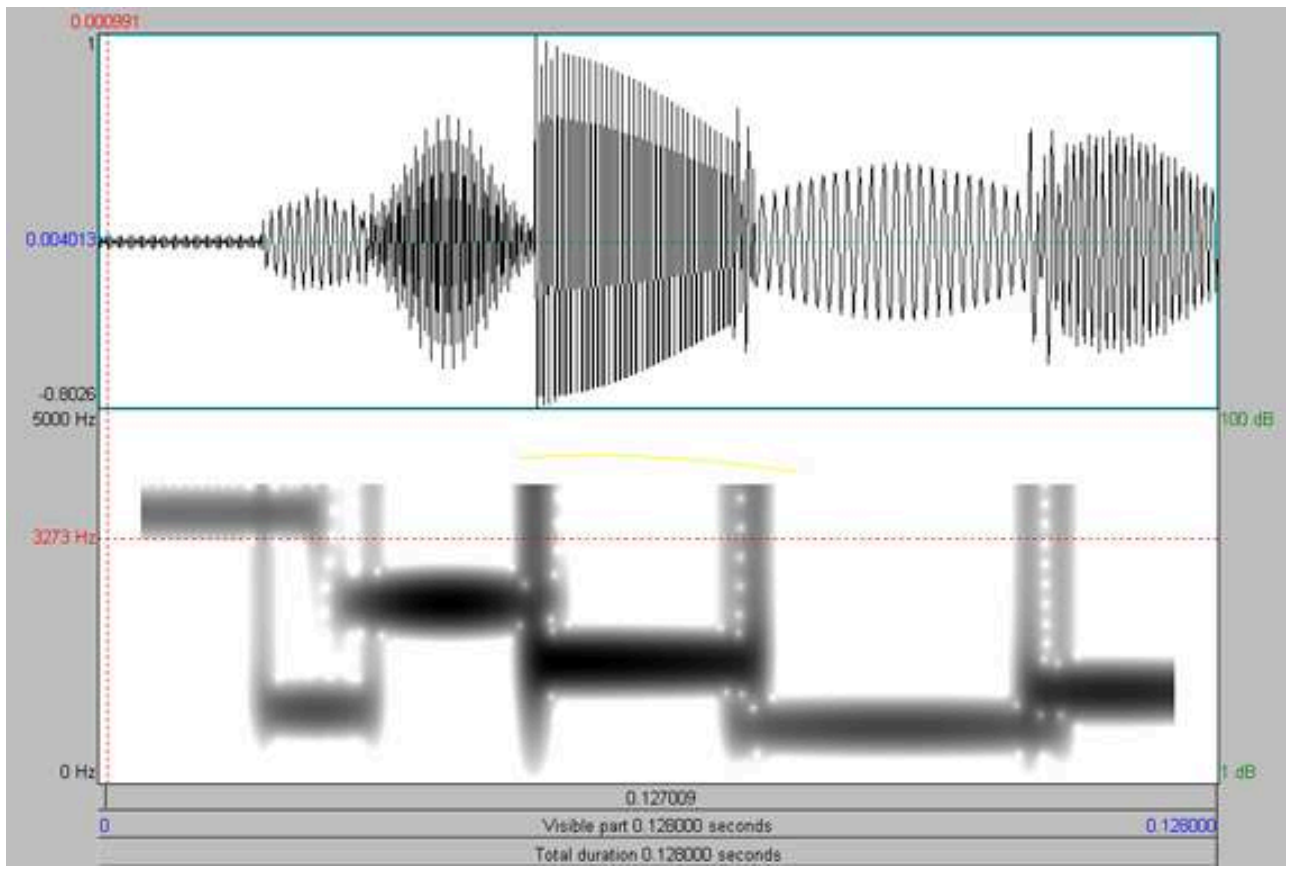

51 La figure précédente illustre l'analyse par Praat de ce signal synthétique. Nous proposons en comparaison l'analyse fournie par notre logiciel. 
Figure 11

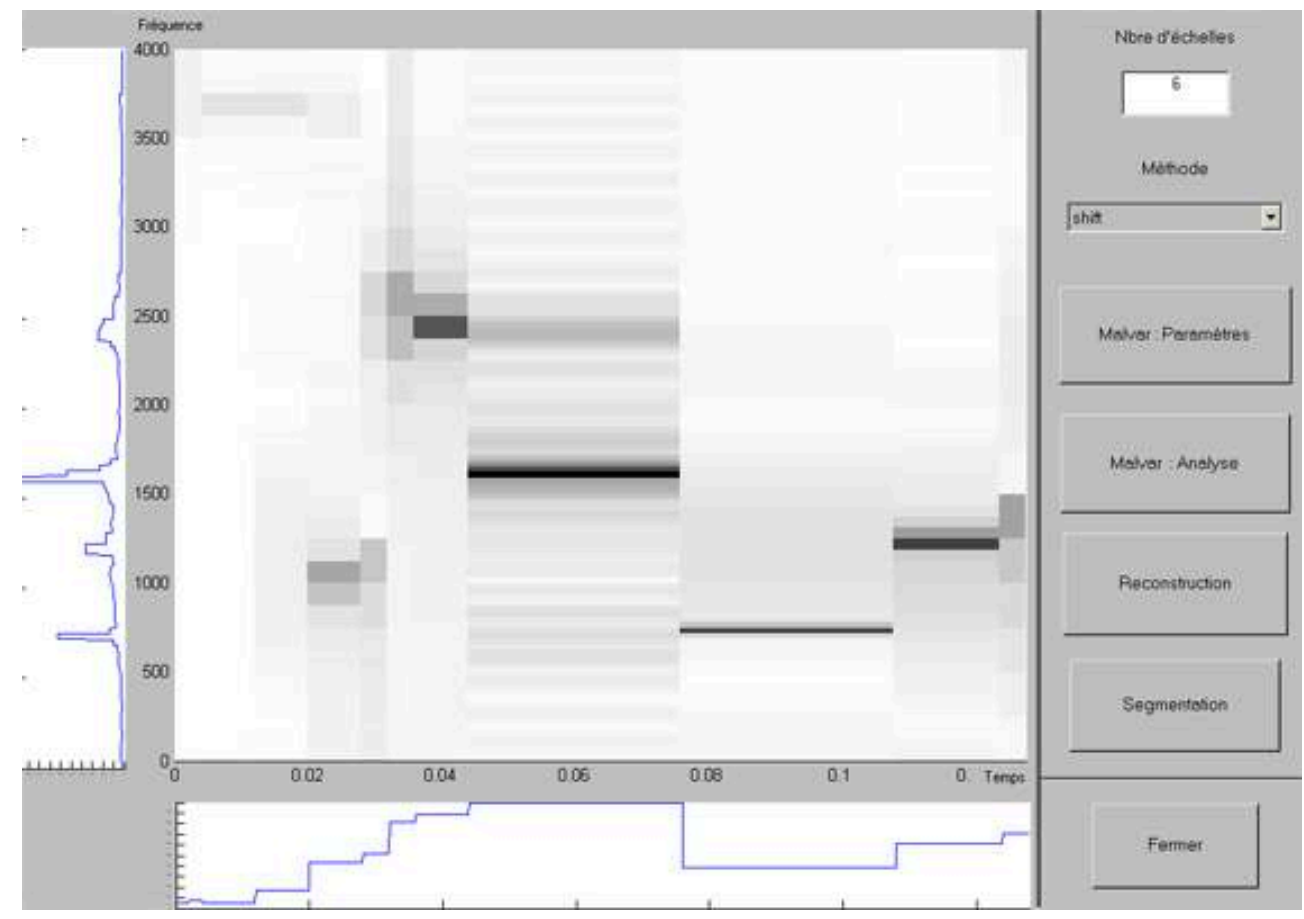

52 On observe facilement les différences, à savoir une traduction fine des fréquences élémentaires présentes dans le signal, alors qu'avec Praat la représentation est plus grossière. Cela s'explique, comme nous l'avons déjà dit plus haut, par un découpage adaptatif, en fonction des éléments du signal. Nous pouvons aussi noter deux courbes complémentaires. La première, sous le spectrogramme, correspond à une mesure de l'énergie présente dans le signal, par blocs homogènes. Cette courbe nous alertera donc sur la présence de pics énergétiques présents dans le signal et nous permettra de repérer la présence de phénomènes accentuels. L'autre courbe, à gauche du spectrogramme, correspond à une mesure d'énergie par fréquence.

Enfin, l'un des avantages de cette famille de méthodes réside dans l'inversibilité des opérations proposées, autorisant une reconstruction locale du signal dans une bande temporelle et fréquentielle donnée. Cette reconstruction locale permet une analyse et l'écoute d'une «brique» particulière du signal analysé. Nous illustrons cette fonctionnalité à l'aide de la figure ci-dessous : 
Figure 12

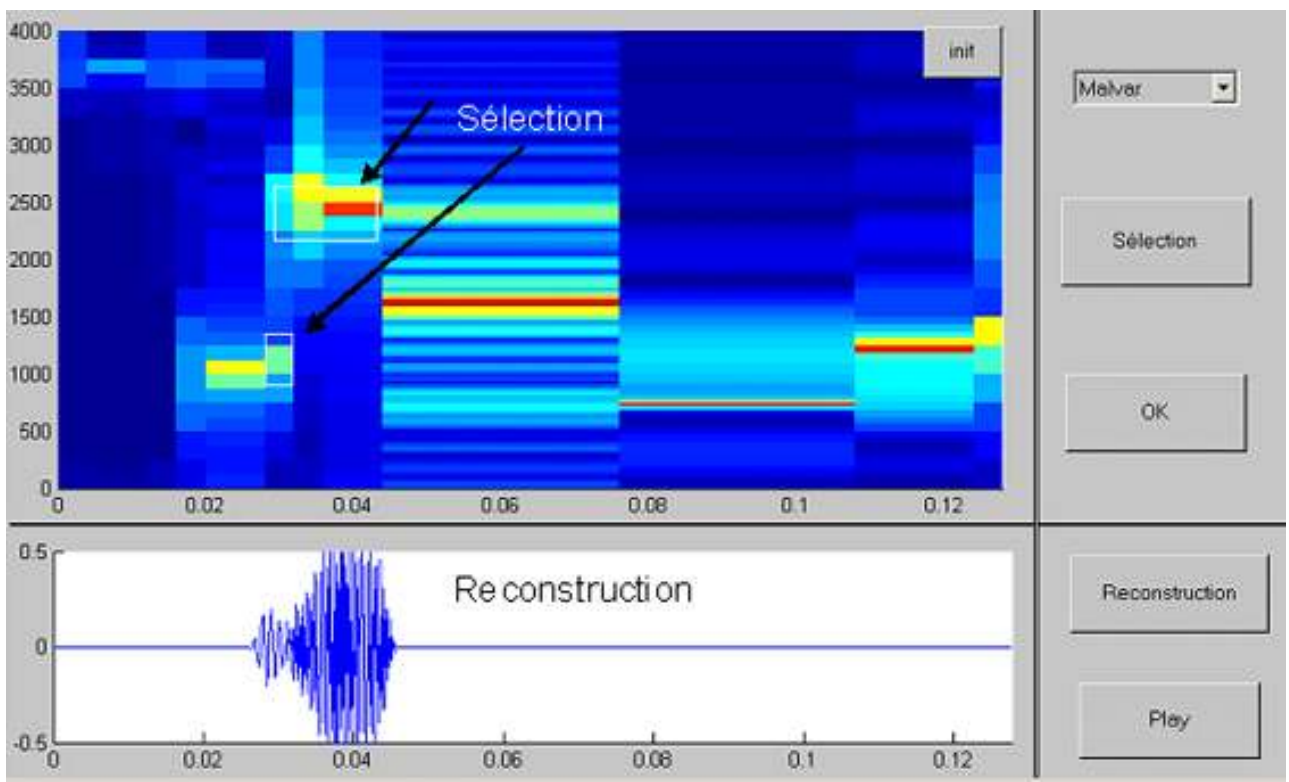

Si cette dernière fonctionnalité peut s'avérer extrêmement intéressante pour des travaux ultérieurs, nous ne l'utiliserons cependant pas dans cette étude car là n'en est pas l'objet. Nous nous concentrerons plus spécifiquement sur les résultats de SWA en ce qui concerne le degré d'accentuation de telle ou telle syllabe dans un mot et la valeur de la voyelle.

\section{Préfixés en discours : analyse acoustique de la prononciation des deux préfixes étudiés un- et de-}

Reprenons les critères définitoires de prononciation des préfixes séparables, proposés en introduction, et dont on a vu le problème de représentation dans notre dictionnaire de référence :

1. tout préfixe séparable est accentué;

2. tout préfixe séparable a une forme pleine ([di: $]$ pour de-, $[\Lambda \mathrm{n}]$ pour un-).

Dans cette troisième partie, nous testerons ces critères sur des fichiers-son qui, comme nous l'avons souligné dans notre deuxième partie, peuvent être issus: 1) du dictionnaire de référence LPD3;2) de documents authentiques (empruntés à BBC Radio 4 ou NPR; 3) de lectures faites par des locuteurs anglophones enregistrés dans des conditions expérimentales.

Associé aux critères accentuels et segmentaux précisés ci-dessus, nous émettons l'hypothèse qu'en discours, il pourrait y avoir d'autres marques de mise en valeur des préfixes séparables, comme la présence d'une rupture dans le flux de parole (pause), la présence d'un coup de glotte à l'attaque du préfixe, en tout cas l'absence de liaison avec les éléments du discours proches (à droite ou à gauche). 


\section{Le préfixe un-}

\section{de l'énoncé suivant :}

(1) The problem seems to be that the test is on computer unfamiliar to students used to a lifetime of paper exams. (Kim Catcheside, BBC News education correspondent)

Fichier audio « Unfamiliar contexte » : à écouter à la fin de cet article ...

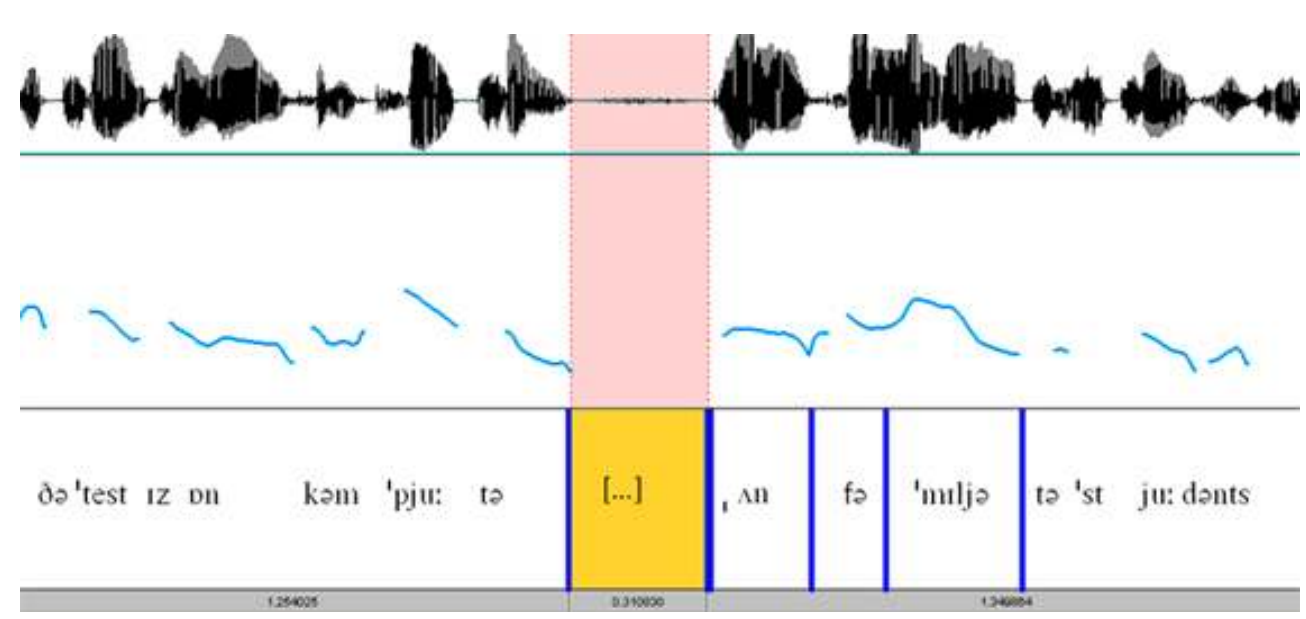


laquelle Nick Leeson (trader britannique qui a causé, du fait de ses manipulations boursières et comptables, la ruine de la plus prestigieuse banque anglaise, la Barings) est interviewé. Le mot qui nous intéresse unscathed est situé à la fin de l'extrait :

(2) John Humphreys: Have you got through it all?

Nick Leeson: I think I've got through that, I think I'm experiencing different things and I've different trials and tribulations all of the time but...I think I've come through that one, relatively unscathed, shall I say. (On the Ropes BBC Radio 4 8/06/04: Nick Leeson)

Fichier audio « Unscathed contexte » : à écouter à la fin de cet article ...

Figure 14

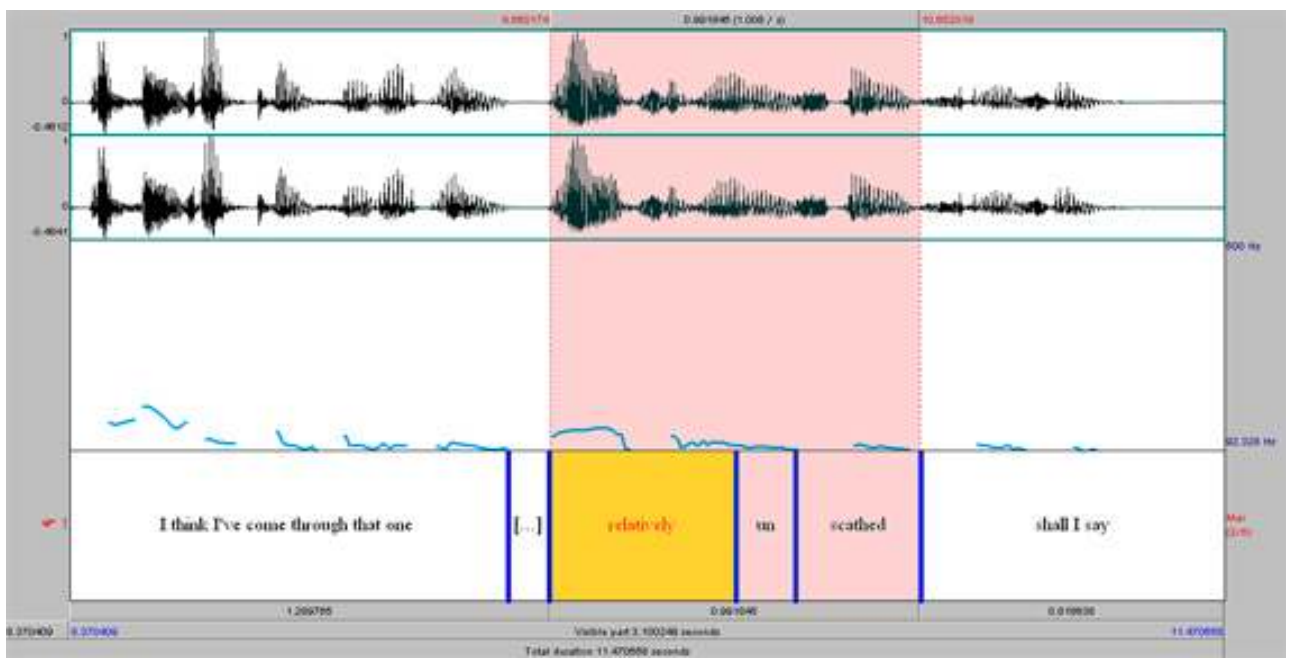
unscathed, où l'adverbe relatively modifie l'adjectif. Il y a dans cet exemple-ci également une pause, mais celle-ci est placé avant le syntagme relatively unscathed. A l'oral, le syntagme relatively unscathed est prononcé dans un seul groupe de souffle. En effet, il y a une montée dans la hauteur de voix sur la première syllabe (syllabe accentuée) de relatively mais le syntagme relatively unscathed est ensuite produit dans un seul groupe de souffle, sans attaque sur l'adjectif, le tout prononcé sur un ton bas. Notons que la fin de l'énoncé shall I say est également prononcé sur un ton bas, sorte de parenthèse, d'incise, postposée. Ainsi, dans cet énoncé, il ne semble pas que le préfixe de l'adjectif unscathed soit particulièrement mis en valeur. Ceci peut sans doute s'expliquer par le fait que l'adjectif scathed n'existe pas (même si l'adjectif scathing existe). De ce fait, il n'y a pas de "concurrence » lexicale possible et il ne peut pas y avoir de mauvaise interprétation de l'énoncé. Par ailleurs, unscathed n'a pas à être opposé à scathed, puisque ce dernier est absent du lexique de l'anglais. Le préfixe n'a donc pas à être mis en valeur et le syntagme relatively unscathed semble fonctionner comme une collocation avec un stress shift sur la première syllabe du syntagme et un accent secondaire sur la base de unscathed, soit le schéma accentuel suivant : /100002/. 
Figure 15

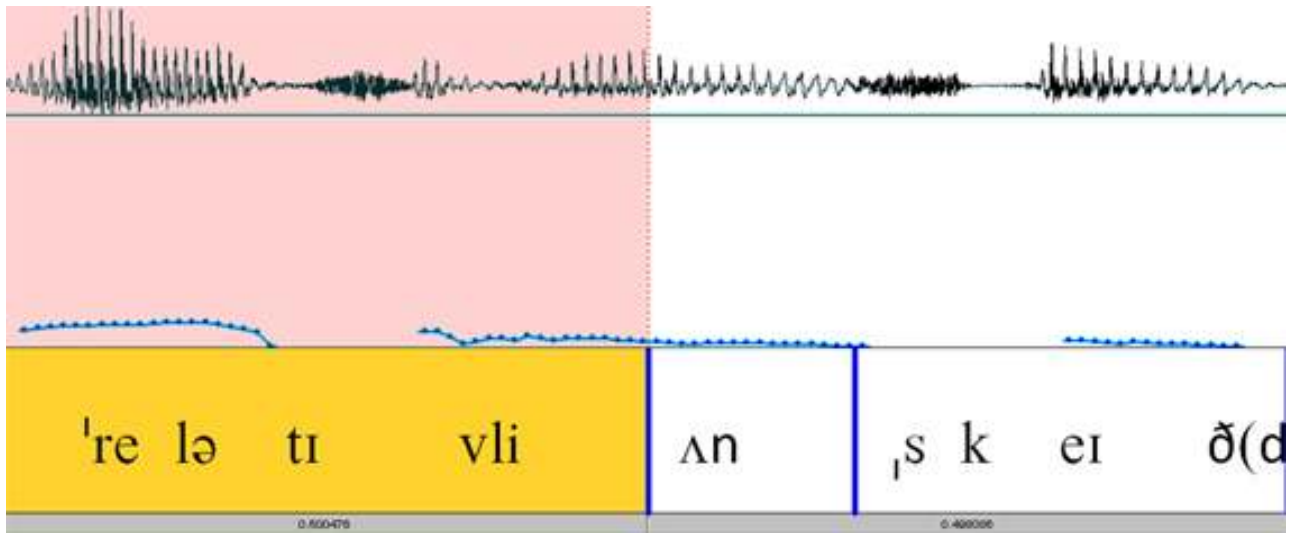

Si l'on prend maintenant le même mot 'unscathed_LPD.mp3' dans le dictionnaire de prononciation LPD3 :

Fichier audio « Unscathed LPD » : à écouter à la fin de cet article ...

Figure 16

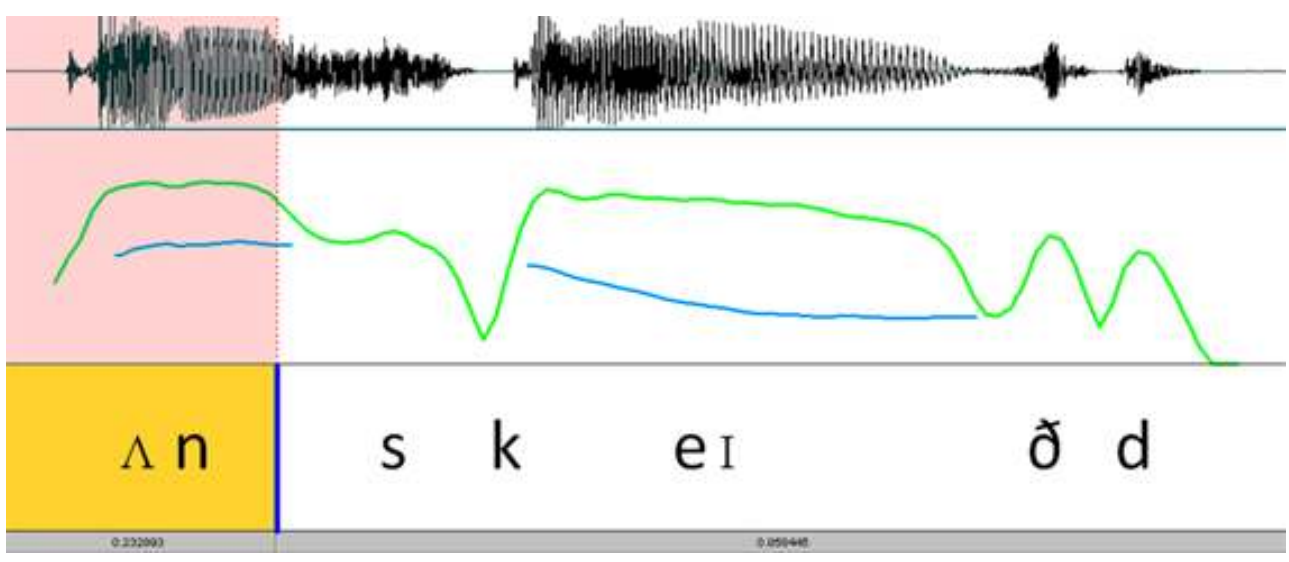

On voit que le préfixe est accentué : il y a en effet une attaque dans la hauteur de la voix (courbe bleue) et une intensité importante sur le préfixe (courbe verte). La notion de préfixe est alors ré-activée. Cela se voit également dans la figure ci-dessous, issue de SWA : 
Figure 17

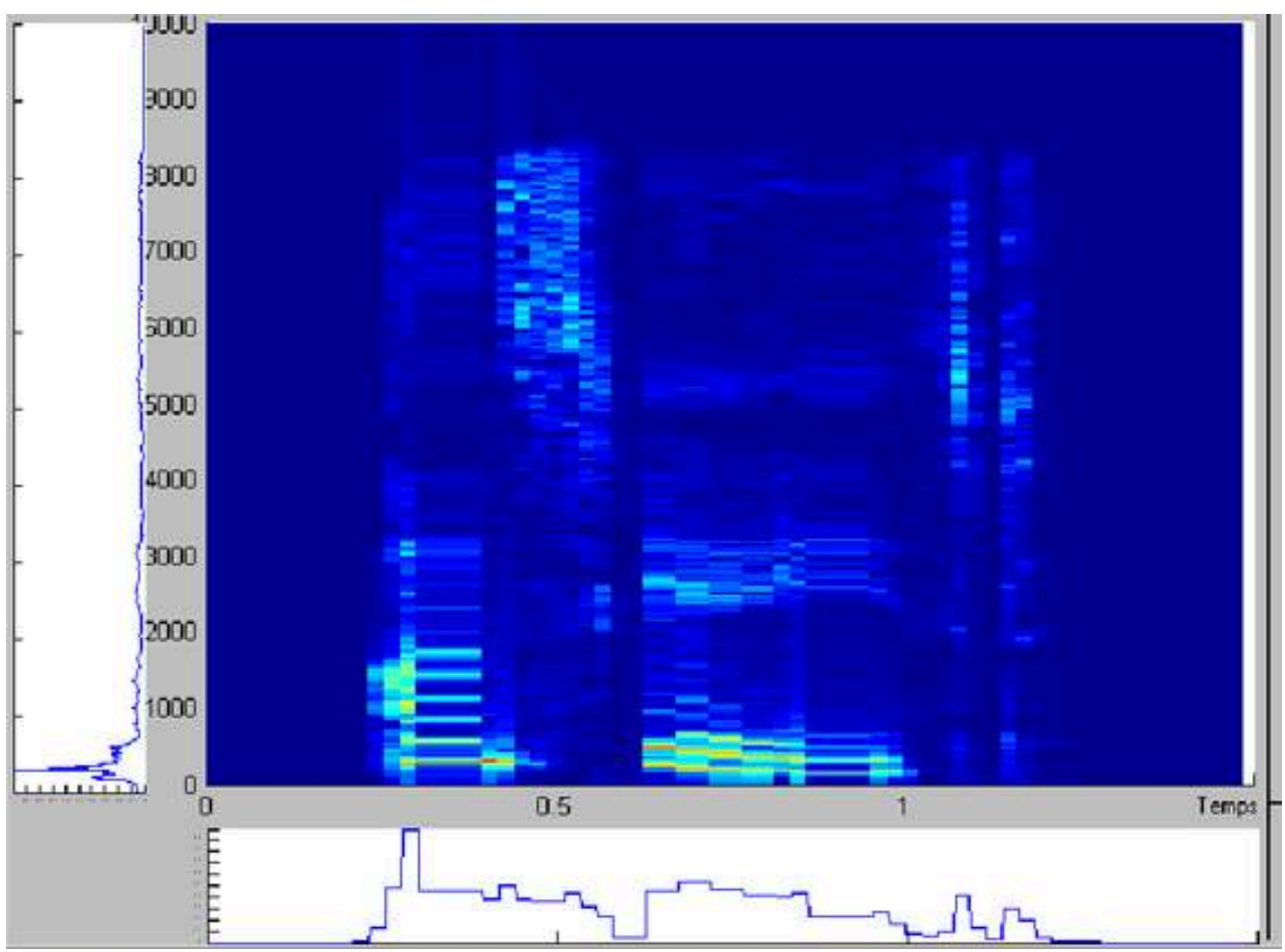

Où l'on a une forte énergie sur le préfixe. La présence d'un accent fort sur le préfixe dans le mot isolé est sans doute liée au fait, justement, de l'absence de contexte linguistique et de la situation artificielle de la lecture mot à mot dont nous avons parlé en 2.1.

En tout cas, dans le cadre de notre énoncé, où le mot est mis en contexte, la préfixation n'est pas particulièrement mise en valeur. On peut même se demander si l'on a encore une forme pleine du préfixe $[\Lambda \mathrm{n}]$, comme cela est noté dans le dictionnaire $\left(\left[()_{1}\right) \Lambda \mathrm{n}\right.$ 'skeıðd]) ou une forme réduite du préfixe ([ən]) :

Figure 18

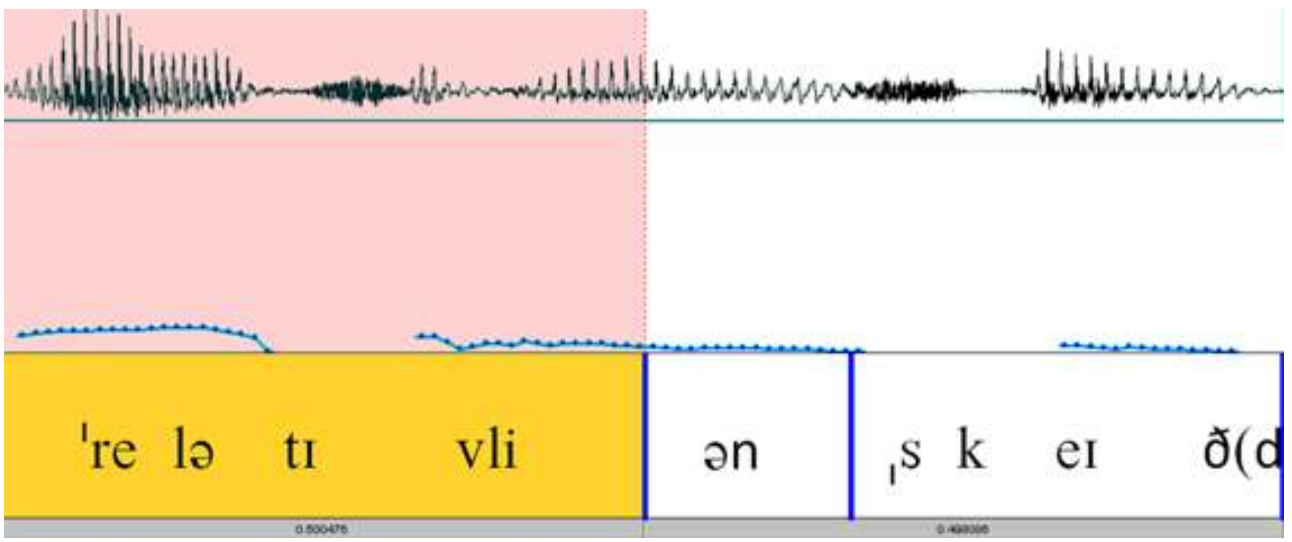

Prenons un dernier exemple. Il s'agit du mot unnatural pour lequel les restrictions rencontrées pour les analyses précédentes sont évacuées (il n'y a en effet pas de contraintes rythmique entraînant l'accentuation du préfixe, et le dérivant natural existe en anglais). 


\section{référence:}

Fichier audio « Unnatural LPD » : à écouter à la fin de cet article ...

Figure 19

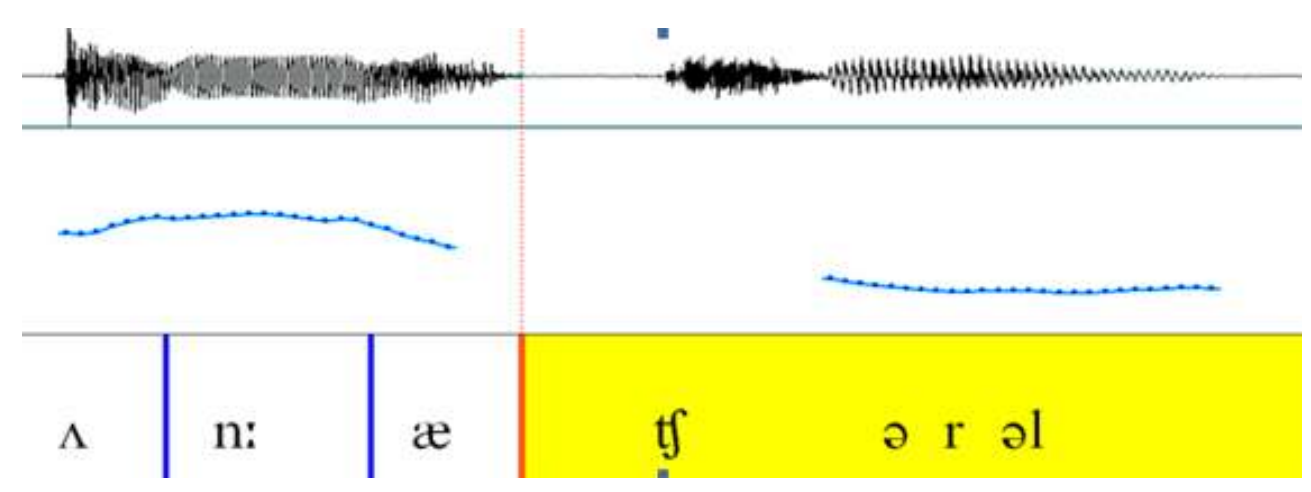

Figure 20

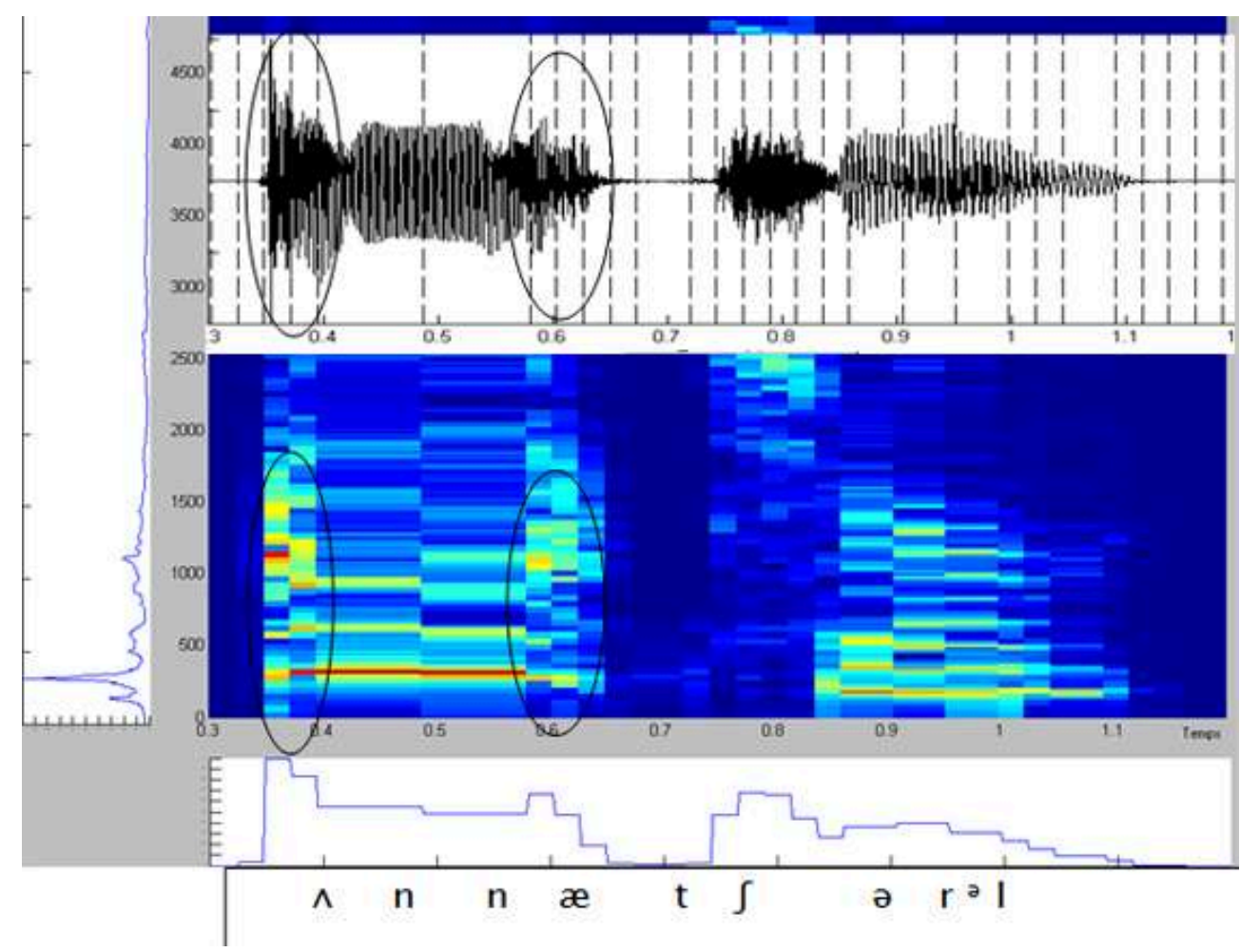

On voit que l'on a une forte énergie sur le préfixe un-, presque même plus intense que sur la syllabe qui porte l'accent principal. On a donc bien ici un préfixe accentué et qui a une forme pleine.

Si l'on fait maintenant l'analyse spectrographique du même mot, mais cette fois-ci en contexte dans le syntagme a little unnatural, issu de l'énoncé suivant:

(3) Sarah: And what do you think about this suggestion of making it illegal to give children under the age of 15 alcohol within the home, which is possible at the moment? 
Sharon: I think that - um, I think that's a little unnatural when um the children see alcohol being drunk in moderation in the family [...]

Fichier audio « Unnatural contexte » : à écouter à la fin de cet article ...

On obtient la représentation suivante:

Figure 21

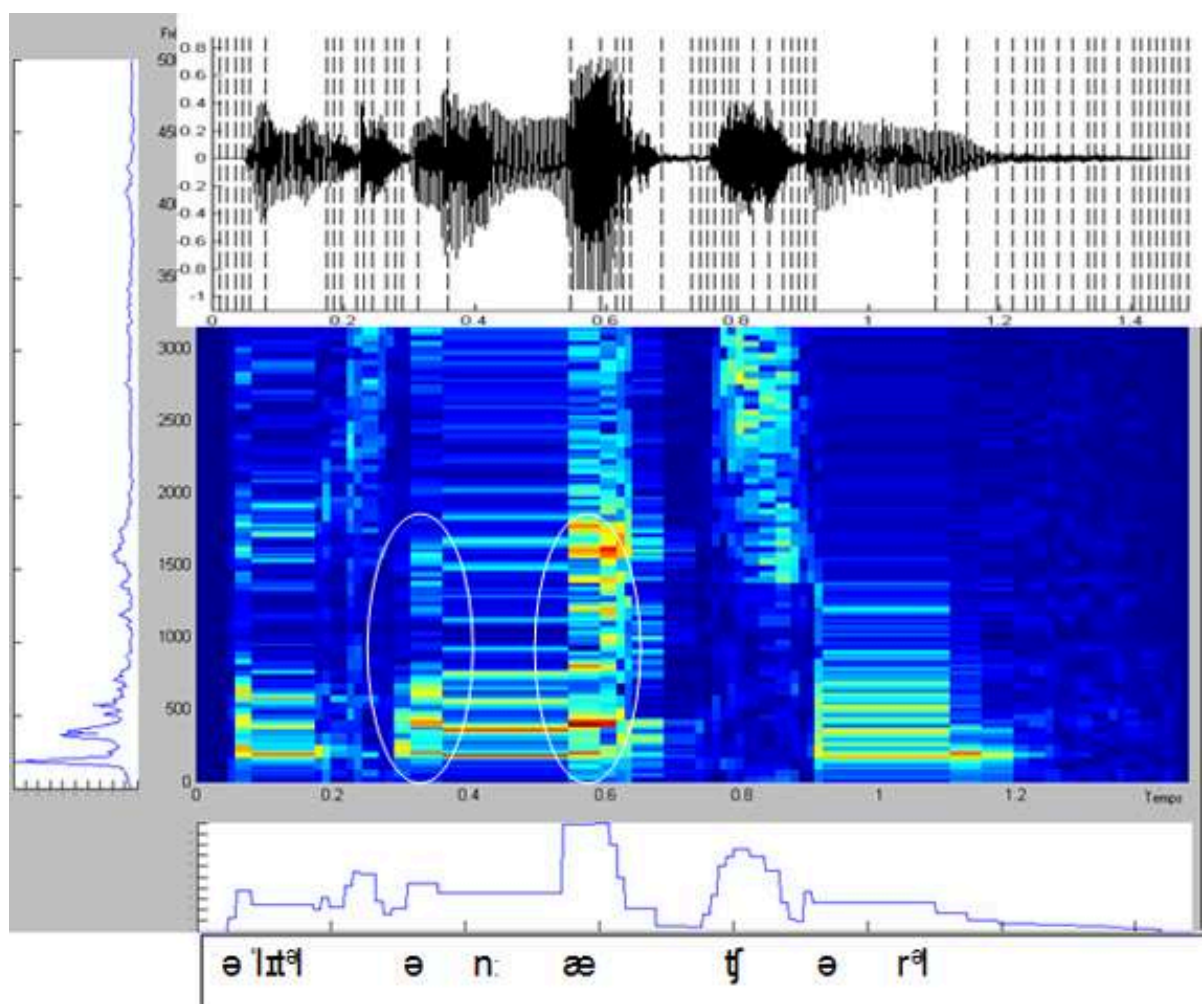

Ce qui est notable ici, notamment dans la reconstruction du signal en blocs énergétiques ci-dessus, c'est le fait que la syllabe qui « absorbe » toute l'énergie est ici la deuxième syllabe du mot <-na->, ce qui est justifié puisqu'elle porte l'accent principal. Par contre, le préfixe est peu accentué, d'où, sans doute, la mise entre parenthèses de l'accent secondaire sur le préfixe dans LPD3: $\left(_{1}\right) \Lambda n$ 'næt $\int$ ər。əl. Ici, contrairement à l'exemple précédent, l'adjectif natural existe, mais on ne peut pas avoir en anglais a little natural ; le risque d'ambiguïté est donc nul, d'où, sans doute, le quasieffacement vocalique du préfixe. En effet, si l'on fait un zoom sur le préfixe, on s'aperçoit que la voyelle du préfixe n'est pas particulièrement présente : 


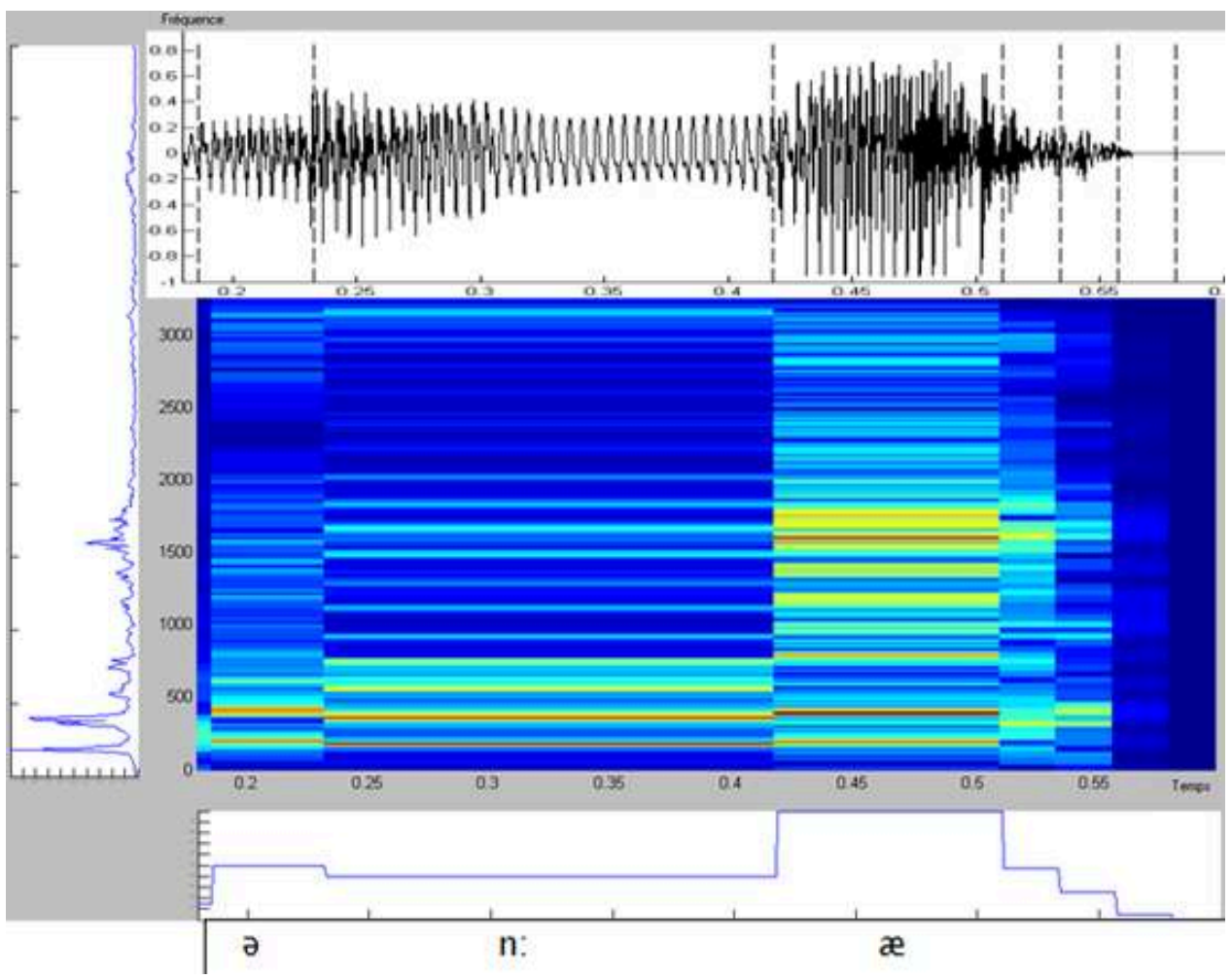

On voit d'ailleurs la différence avec les mots précédents (notamment unnatural (LPD3), ou unfamiliar) dans lesquels on avait une énergie plus importante sur le préfixe et où l'on avait les formants définitoires de la voyelle pleine $[\Lambda]$ ).

Pourrait-on aller jusqu'à émettre l'hypothèse que l'on a ici pas de voyelle mais simplement un [n] syllabique suivi d'un [n] d'attaque syllabique? Cela est possible. Notons quand-même que SWA propose un découpage entre les deux zones, ce qui signifierait que l'on a quand-même une présence vocalique (présence d'un [ə])? Le [1] syllabique de little serait ainsi suivi d'un préfixe peu accentué (voyelle très courte [ə] voire [n] syllabique). Il n'y a ni pause avant ou après le préfixe, ni accentuation particulière du préfixe pour le mettre en valeur. Cela peut-il occasionner une gêne dans l'interprétation de l'énoncé par l'auditeur? Sans doute pas car, d'une part, le contexte sémantique général va pouvoir aider l'auditeur à traduire l'information (même si l'on n'a pas la prononciation d'un préfixe plein dans la chaîne phonique), d'autre part, l'information est ici sans doute codée dans la longueur de la consonne du préfixe (le [n:] de unnatural). Cependant, il serait sans doute important de revenir sur l'enseignement de la préfixation en anglais, où il est fréquent de présenter les préfixes séparables comme porteur d'un accent et ayant une forme pleine:

It is important to distinguish between the secondary stress of prefixes, such as , rein ,re-'act or ,inter- in ,inter'national, and the secondary stress in words, such as inter'vention or ,kanga'roo for example: while the secondary stress of prefixes expresses their lexical status, the secondary stress of these words is due to the [...] impossibility of beginning an English word with two unstressed syllables; neither inter, in the inseparable prefixed, or, obviously, kang(-) in kangaroo, have lexical status. (Deschamps, A. et al. $2004: 44$ )

D’après les quelques fichiers-son présentés ici, les hypothèses présentées en début de partie sur la mise en valeur du préfixe en cas de séparabilité de celui-ci ne sont pas 
véritablement vérifiées en discours sauf, sans doute, en cas de mise en valeur contrastive. C'est ce que nous allons voir maintenant avec le préfixe de-.

\section{Préfixe de- : cas de decelerate}

Pour le préfixe de-, nous nous attacherons essentiellement à l'étude du verbe decelerate, qui, nous l'avons vu, enfreint les règles établies par le cadre théorique utilisé : la base est non autonome mais le préfixe demeure porteur d'un accent secondaire et d'une voyelle tendue.

Commençons par l'étude sous Praat de l'extrait sonore du LPD3 (voix féminine; prononciation britannique) :

Fichier audio « Decelerate LPD » : à écouter à la fin de cet article ...

Figure 23

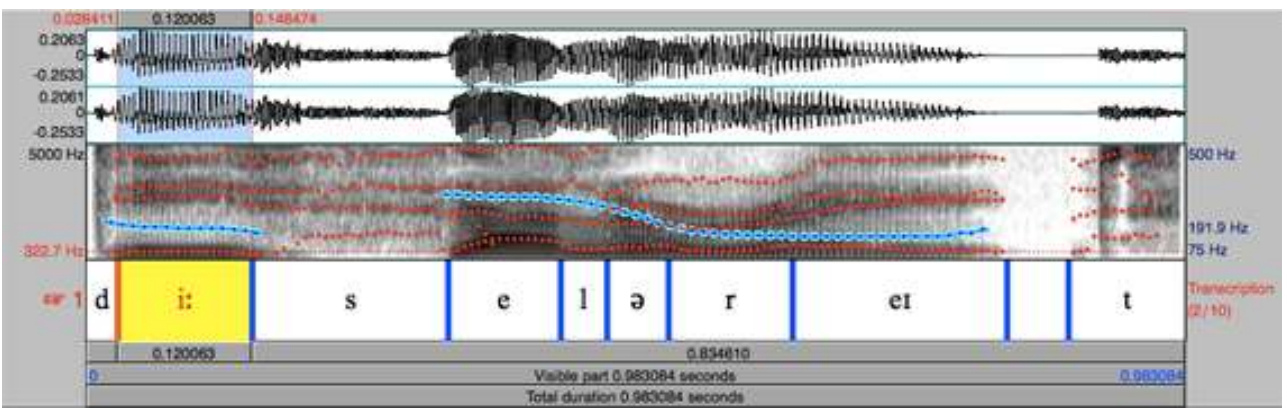

Le spectrogramme obtenu indique clairement que la voyelle du préfixe est longue. Sa longueur avoisine $0,12 \mathrm{~s}$. Son $1^{\mathrm{er}}$ formant a une fréquence relativement basse $(322 \mathrm{~Hz})$ et le $2^{2 \text { ème }}$ formant est élevé $(2676 \mathrm{~Hz})$. Ses 2 fréquences sont définitoires d'une voyelle plutôt tendue, ce qui correspond bien à la transcription donnée par LPD.

Bien qu'il s'agisse de la prononciation la plus couramment obtenue, certains locuteurs enregistrés pour le corpus lecture ont prononcé ce verbe avec un préfixe inaccentué et doté d'une voyelle courte, prononciation qui, rappelons-le, n'est pas proposée en variante dans $L P D$. En voici un exemple, avec l'énoncé suivant:

(4) It takes a measurable time for the motor to decelerate to the point where the clutch disengages and, during this time, it will tend to slow the rotor, which is undesirable. (voix féminine ; anglais britannique standard).

Fichier audio « Decelerate contexte " : à écouter à la fin de cet article ...

Figure 24

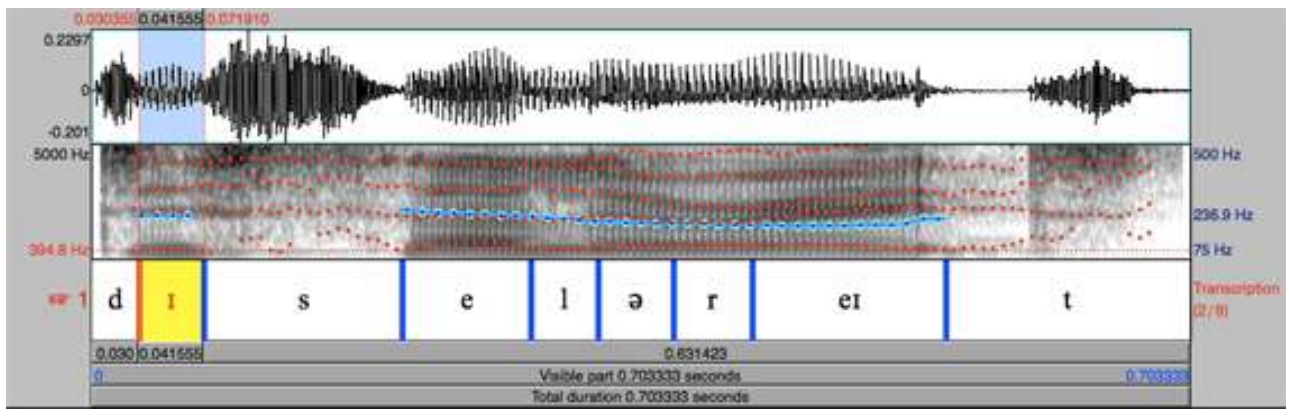


Figure 25

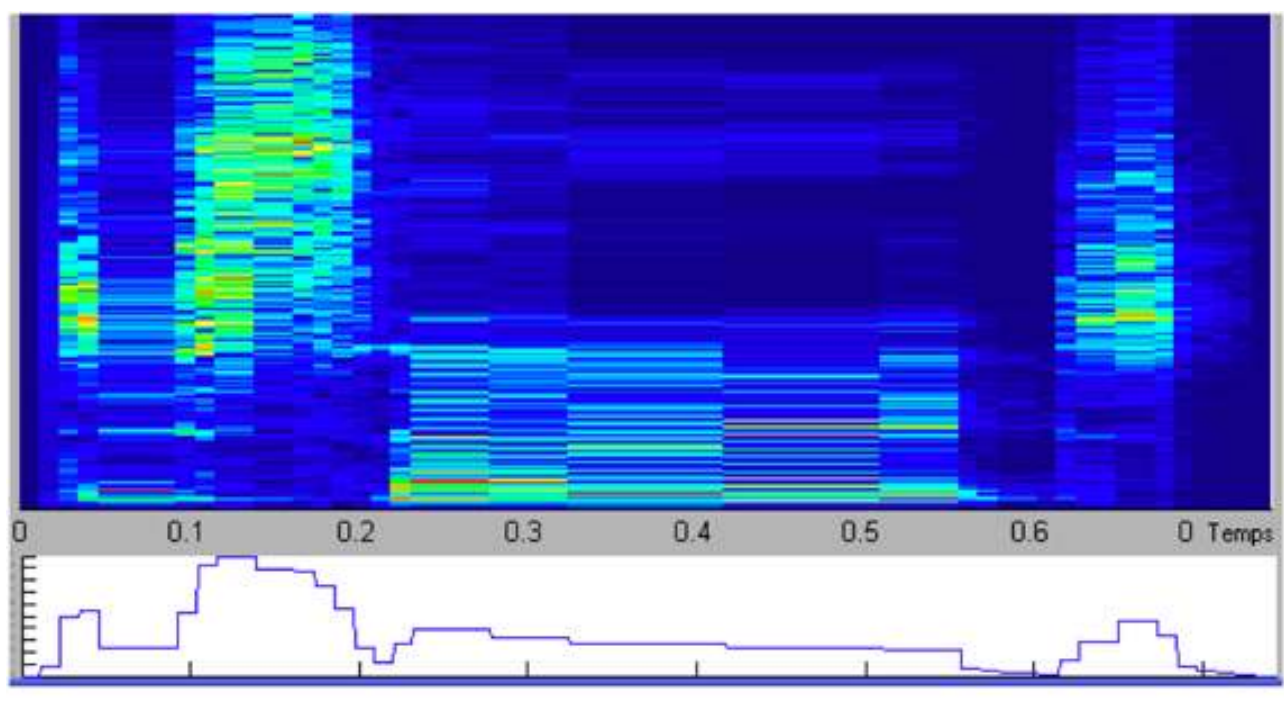

d $\quad$ I S

$\mathrm{e}$

lo

reI

$\mathrm{t}$

82 Le spectrogramme obtenu sous Praat montre que la voyelle du préfixe est ici très courte $(0,04 \mathrm{sec})$ avec des formants caractéristiques d'un [I] (F1 $=394 \mathrm{~Hz}$ et F2 $=2075 \mathrm{~Hz})$. De plus, comme on peut l'observer sur la figure obtenue avec Speech Wave Analyser, il y a très peu d'énergie sur le préfixe. Toutes ces données mènent à conclure que la prononciation [dI'seləreIt], qui obéit aux règles de la théorie, est tout à fait possible et pourrait par conséquent apparaître en variante dans LPD.

\section{Préfixation et contraste paradigmatique}

83 Dans notre première partie, nous avons avancé que la prononciation telle qu'elle est notée dans LPD pourrait se justifier par le fait que de-entre dans un paradigme avec le préfixe ac- (allomorphe de ad-) dans accelerate. Cette hypothèse nous a amenés à poser la question suivante : est-ce que les locuteurs qui ont produit le mot de cette façon le prononceraient différemment s'il était précédé du verbe accelerate, afin d'exprimer le contraste entre les deux verbes?

84 Afin d'y répondre, nous avons inclus également dans notre corpus lecture un énoncé dans lequel decelerate était associé à accelerate dans le co-texte proche. Voici la prononciation obtenue par la même locutrice que pour l'exemple (4) :

(5) It can provide information regarding the possible need to change management style, to accelerate, decelerate or even terminate the activity earlier and hence save scarce resources which can be used to good effect elsewhere. (voix féminine; anglais britannique standard).

Fichier audio « Decelerate 2 contexte » : à écouter à la fin de cet article ... 


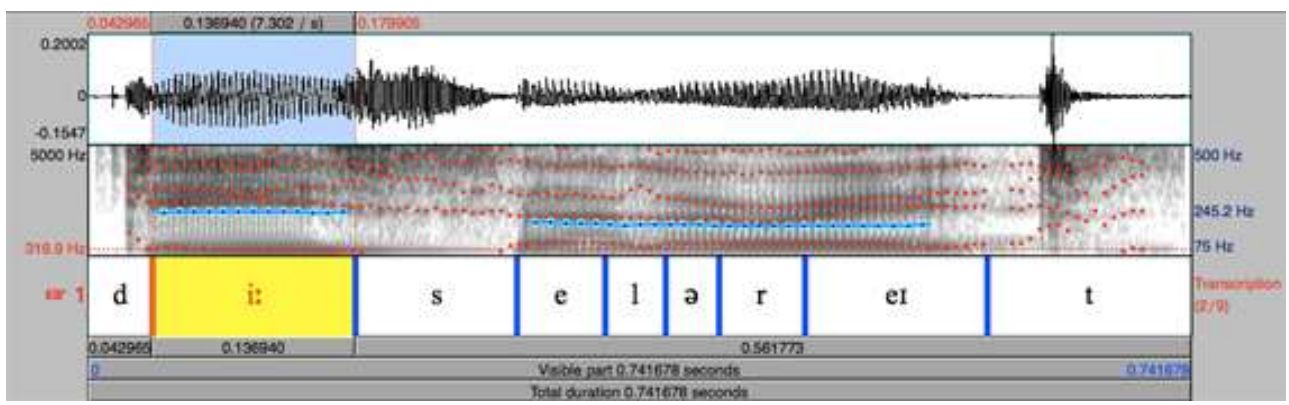

Figure 27

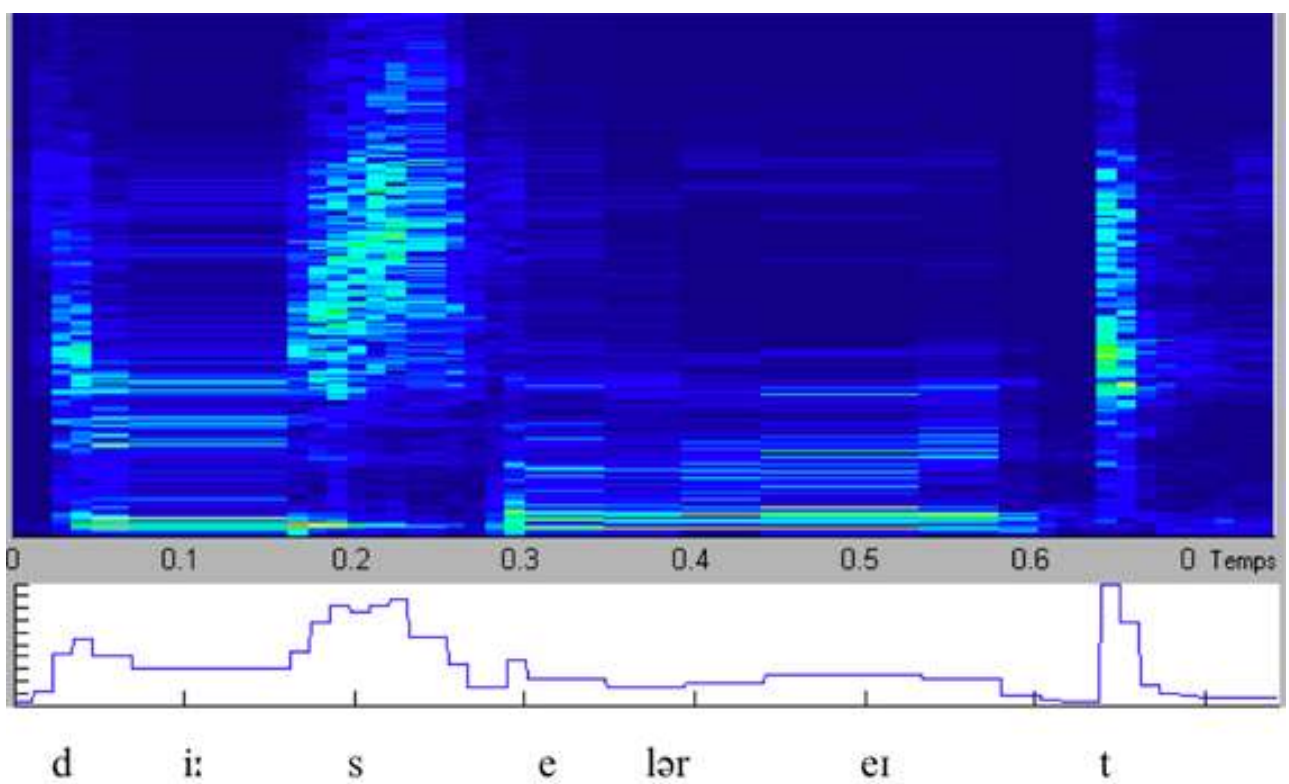

L'hypothèse est confirmée puisque la prononciation de decelerate dans ce contexte, et par la même locutrice que précédemment, est clairement différente. On constate un net allongement de la voyelle $(0,13 \mathrm{sec})$ et des fréquences $\mathrm{F} 1$ et $\mathrm{F} 2$ définitoires d'une voyelle tendue $(320 \mathrm{~Hz}$ et $2500 \mathrm{~Hz})$. Quant à l'accentuation du préfixe, on observe un bloc d'énergie homogène assez élevé durant toute la tenue de la voyelle, qui témoigne de la présence d'un accent. L'hypothèse de la proximité syntaxique du verbe accelerate déclenchant une telle prononciation du verbe decelerate est donc confirmée.

S'ensuit le questionnement subsidiaire : cet effet de contraste paradigmatique, donc d'accentuation du préfixe et de changement vocalique, apparaît-il essentiellement sur le deuxième élément de la paire? Autrement dit, si decelerate a été affecté parce qu'il apparaissait après accelerate dans l'énoncé, que se passerait-il si les deux termes se trouvaient inversés?

Soit l'exemple suivant, issu d'une émission radiophonique de la radio américaine NPR :

(6) It would be very difficult to claim that Iraq was stepping up its unconventional weapons programs cause in fact Sadam Hussein was decelerating that effort rather than accelerating it. (voix masculine ; anglais américain, NPR).

Fichier audio « Accelerating contexte " : à écouter à la fin de cet article ... 


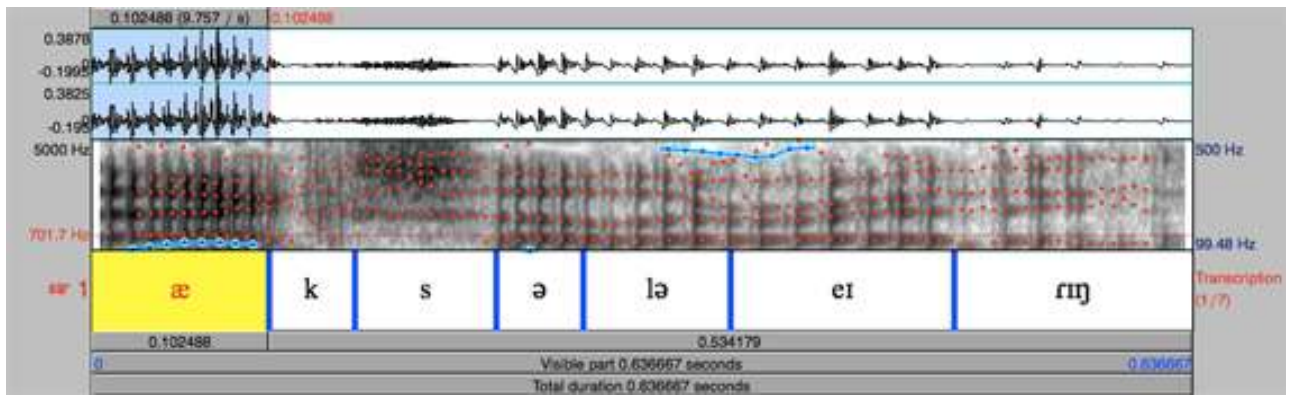

Figure 29

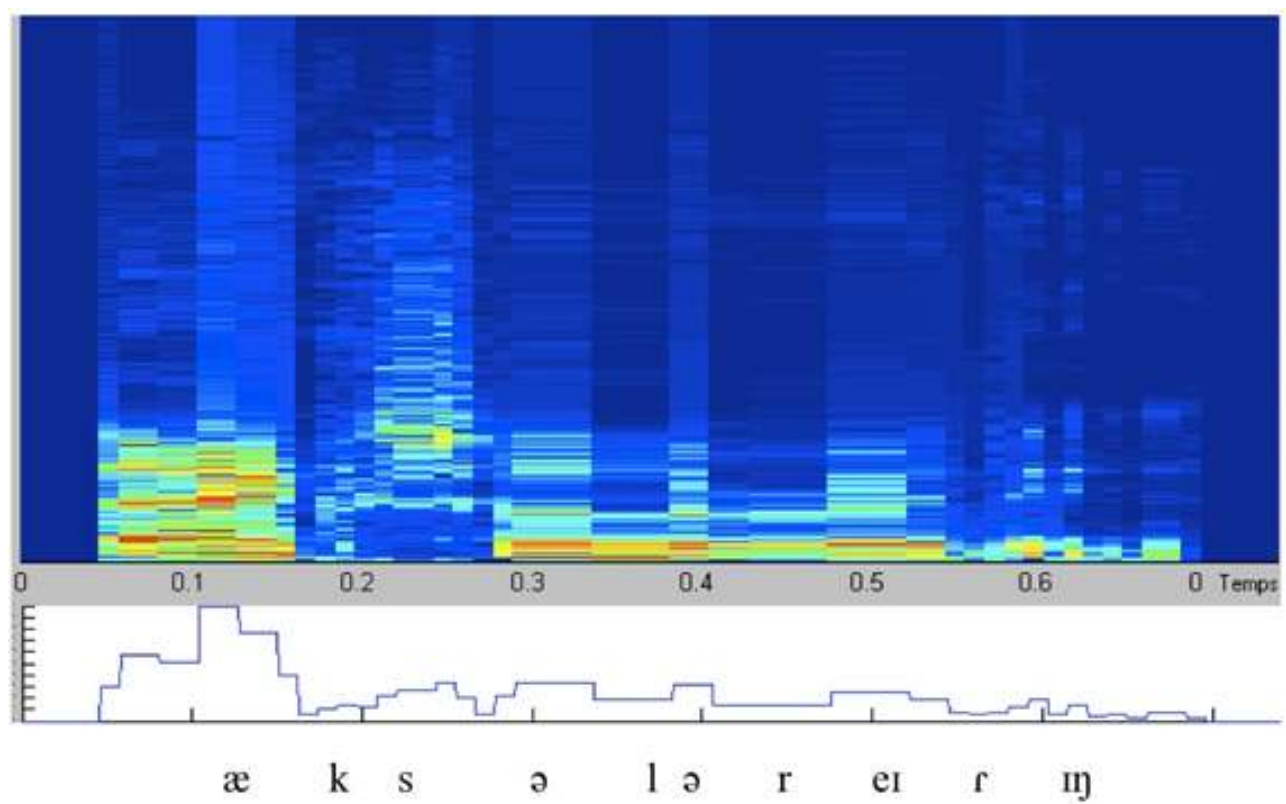

Le spectrogramme obtenu sous Praat montre en effet que la prononciation de la voyelle du préfixe ac- est modifiée. Il s'agit ici d'une voyelle pleine, ayant pour fréquence de formants $\mathrm{F} 1=701 \mathrm{~Hz}$ et $\mathrm{F} 2=1701 \mathrm{~Hz}$. Ces fréquences sont caractéristiques de la voyelle [æ]. Cette prononciation du préfixe correspond à la prononciation qui apparaît en variante dans LPD. La surprise vient du schéma accentuel de ce verbe. La figure donnée par Speech Wave Analyser montre un niveau d'énergie très élevé sur la voyelle du préfixe, témoignant d'un schéma /10000/. De plus, il semble que la voyelle de la première syllabe de la base (normalement [e]) soit réduite à [ə]. On aurait donc la prononciation ['æksələreIrıy]. Cette prononciation n'est absolument pas prévue par $L P D$. Elle va même à l'encontre des règles d'accentuation lexicale de l'anglais : -ate, terminaison contraignante, impose l'accent sur l'antépénultième. On peut donc en conclure que cet effet de contraste, associé à la charge sémantique des préfixes, peut prendre le pas sur certaines règles d'accentuation fortes (en l'occurrence ici, celle des mots en -ate). 


\section{Conclusion} surtout du contexte:

- «contexte " lexical: dans le cas de unscathed par exemple, nous avons vu que le préfixe n'était pas accentué en phrase, qu'il était sans doute même réduit. Nous avons noté que c'était sans doute lié au fait qu'il n'y avait pas de concurrence lexicale possible; scathed n'est en effet pas attesté en anglais contemporain. Il n'y a ainsi pas de confusion possible.

- contexte syntaxique: relatively unscathed dans notre exemple (2) peut être envisagé comme une collocation, l'adverbe + adjectif forment un ensemble accentuel, prononcé dans un seul groupe de souffle, sans doute avec un stress-shift sur la première syllabe de l'adverbe, d'où l'absence d'accent sur le préfixe. Il se passe la même chose dans l'exemple (3), dans lequel le syntagme a little natural ne semble pas possible, d'où le quasi-effacement du préfixe un-alors que natural, contrairement à scathed, est attesté en anglais.

- contexte discursif: dans les exemples (5) et (6), c'est la présence de la paire accelerate et decelerate dans le même énoncé qui provoque sans doute la prononciation pleine et marquée du préfixe. Il s'agit-là de contraste paradigmatique. Il y a volonté de la part du locuteur de contraster deux éléments lexicaux antonymes. Dans ce cas-là, le préfixe subit une « recharge sémantique » et est de ce fait accentué. Il s'agit d'emphase.

91 Il resterait maintenant à tester le contexte phonétique, et bien d'autres sans doute encore. Tous ces paramètres devront également être testés sur un corpus plus important car, pour pouvoir proposer des hypothèses plus larges sur la prononciation des préfixes en contexte, il sera nécessaire de travailler sur un corpus oral de grande taille, et vérifier nos hypothèses sur la prononciation en contexte d'autres préfixes de l'anglais.

BIBLIOGRAPHIE 


\section{Ouvrages / Etudes théoriques}

ANDREWS, E. (1986). « A Synchronic Semantic Analysis of de- and un- in American English ».

American Speech, vol. $61 \mathrm{n}^{\circ}$ 3. 221-232.

CALlioPe, éd. (1989). La parole et son traitement automatique. Paris: Masson.

CRUTTENDEN, A. (2001 [1962]). Gimson's Pronunciation of English. Sixth Edition. London: Edward

Arnold.

DesChAMPS, A. et al. (2004). English Phonology and Graphophonemics. Gap \& Paris: Ophrys.

FOWLER, H.W. (1996) The New Fowler's Modern English Usage. Oxford: Oxford University Press.

FUDGE, E. (1984) English word-stress. London: Allen \& Unwin

GUIERRE, L. (1979) L'accentuation en anglais contemporain - Eléments pour une synthèse. Paris : Université

Paris 7-Didier.

GUIERRE, L. (1984). Drills in English Stress Patterns, $4^{\text {th }}$ ed. Paris : A. Colin-Longman.

GUIERRE, L. (1987). Règles et exercices de prononciation anglaise. Paris : A. Colin

HANOTE, S. et F. ZUMSTEIN (avril 2008) « Etude phonologique et acoustique des préfixes in- et un- ». $14^{\text {ème }}$ colloque de l'ALOES. Villetaneuse.

PAILLARD, M. et N. VIDEAU. (2008). « Les verbes préfixés en dé- du français et leurs traductions en anglais ». in PAILLARD M. (dir.), Préfixation, prépositions, postpositions. Étude de cas. Rennes: Presses Universitaires de Rennes.

VIDEAU, N. (2008). Le préfixe anglais de-: analyse phonologique et acoustique. TER de Master 2 sous la direction de Mme. S. HANOTE. Université de Poitiers.

ZUMSTEIN, F. (2007). Variation accentuelle, variation phonétique: étude systématique fondée sur des corpus lexico-phonétiques informatisés anglais. Thèse de doctorat. Université de Poitiers.

\section{Dictionnaires}

JONES, D. (1963) Cambridge English Pronouncing Dictionary. $12^{\text {th }}$ ed. Cambridge: Cambridge University Press.

The Oxford English Dictionary (1989). Oxford: Clarendon Press.

The Longman Dictionary of English Language and Culture (1998). Harlow: Longman.

WELLS, J.C. $\left(1990,2000\right.$ et 2008). Longman Pronunciation Dictionary. $1^{\text {st }}, 2^{\text {nd }}$ and $3^{\text {rd }}$ editions. London : Longman.

\section{Corpus radiophonique}

BBC Radio 4 : http://www.bbc.co.uk/radio4/

$N P R:$ http://www.npr.org/ 


\section{NOTES}

1. Catégories syntaxiques systématiquement marquées, indication du schéma accentuel principal (/1/ pour syllabe avec accent principal, /2/ pour syllabe avec accent secondaire, /3/ pour syllabe avec accent tertiaire, /0/ pour syllabe inaccentuée), indication du nombre de syllabes et graphie inverse.

2. Macintosh ${ }^{\circ}$ Programmer's Workshop (http://developer.apple.com/tools/mpw-tools/ pour informations et téléchargement).

3. L'accent primaire tombe sur la seconde syllabe du verbe unquote en anglais britannique, et sur

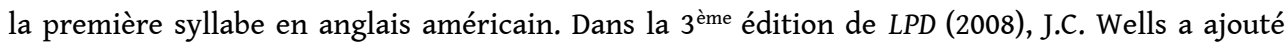
l'adjectif uncount que l'on trouve essentiellement dans l'expression uncount noun et dont la première syllabe reçoit un accent primaire.

4. Dans de telles configurations accentuelles, L. Guierre renvoie à la notion de "conspiration de règles" qui fait de ces exemples des cas «non pertinents » pour l'analyse.

5. Il est important de noter ici que pour tous les mots de cette catégorie, l'accent principal se porte sur la syllabe qui suit directement le préfixe.

6. Surligné par nous.

7. Seuls unfold et uncover ont une variante accentuelle en /21-/.

8. L'adjectif unadvisable semble peu fréquent: nous avons interrogé le British National Corpus (BNC), via l'interface BNCweb (http://www.bncweb.info/) qui propose seulement deux résultats d'occurrences.

9. Les variantes en /21-/ ou /(2)1-/ des adjectifs sont certainement liées à des contraintes discursives : à chacun des adjectifs correspond l'antonyme non préfixé, et le jeu contrastif en discours entraîne l'apparition de l'accent secondaire sur le préfixe. Dans cette hypothèse, les adverbes sont certainement bien moins sujets à une telle variation. De même, au niveau discursif, le «stress shift» (rétraction accentuelle qui obéit à une contrainte contextuelle et rythmique) peut se mettre en place, notamment pour les adjectifs épithètes : Chinese $/ 01 /+$ army $/ 10 /=$ Chinese army /1010/.

10. À l'instar des adjectifs (voir note précédente), la condition d'apparition de la variante est liée à un jeu discursif.

11. En anglais, les mots grammaticaux (auxiliaires, pronoms, prépositions, etc.) ont généralement des formes réduites non accentuées, qui s'opposent aux fromes pleines, formes accentuées. L'usage de l'une ou de l'autre des deux formes est généralement conditionné par des règles discursives.

12. L. Guierre a fondé ses analyses sur une version informatisée par ses soins de la douzième édition (1963) du dictionnaire de Daniel Jones, English Pronouncing Dictionary.

13. À noter que la variante en /(2)1/ n'est pas considérée comme «non-RP ».

14. Voir l'étymologie donnée par OED.

15. Selon $O E D$, crease, aujourd'hui obsolète, signifiait increase. Le mot crease existe en anglais contemporain, mais il n'a rien à voir avec la paire increase/decrease.

16. http://www.fon.hum.uva.nl/praat/ 


\section{AUTEURS}

SYLVIE HANOTE

FORELL A, EA3816, Université de Poitiers

NICOLAS VIDEAU

FORELL A, EA3816, Université de Poitiers

FRANCK ZUMSTEIN

CLILLAC-ARP, EA 3967, Université de Paris 7

PHILIPPE CARRÉ

X-LIM SIC UMR CNRS 6172, Université de Poitiers 